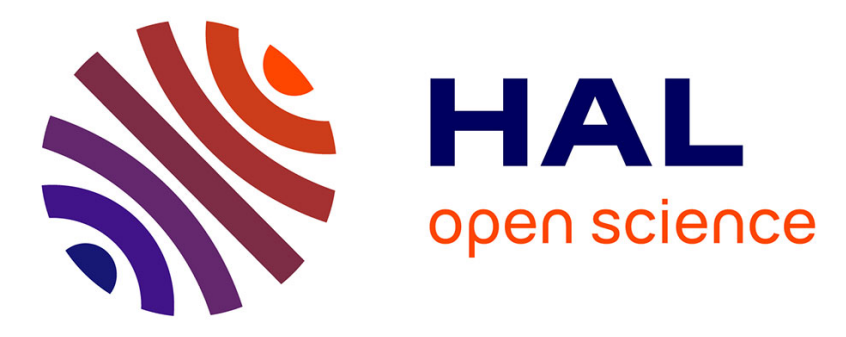

\title{
Observer Design for Nonlinear Systems With Output Transformation
}

\author{
Francisco González de Cossío, Madiha Nadri, P Dufour
}

\section{To cite this version:}

Francisco González de Cossío, Madiha Nadri, P Dufour. Observer Design for Nonlinear Systems With Output Transformation. IEEE Transactions on Automatic Control, 2020, 65 (12), pp.5205 - 5219. 10.1109/tac.2020.2971934 . hal-03401114

\section{HAL Id: hal-03401114 https://hal.science/hal-03401114}

Submitted on 25 Oct 2021

HAL is a multi-disciplinary open access archive for the deposit and dissemination of scientific research documents, whether they are published or not. The documents may come from teaching and research institutions in France or abroad, or from public or private research centers.
L'archive ouverte pluridisciplinaire HAL, est destinée au dépôt et à la diffusion de documents scientifiques de niveau recherche, publiés ou non, émanant des établissements d'enseignement et de recherche français ou étrangers, des laboratoires publics ou privés. 
This document must be cited according to its final version which is published in a journal as:

F. González de Cossío, M. Nadri, P. Dufour, "Observer Design for Nonlinear Systems with Output Transformation ", IEEE Transactions on Automatic Control (TAC), 2020, 65(12), 5205 - 5219, DOI : 10.1109/TAC.2020.2971934

You downloaded this document from the CNRS open archives server, on the webpages of Pascal Dufour: http://hal.archives-ouvertes.fr/DUFOUR-PASCAL-C-3926-2008 


\title{
Observer Design for Nonlinear Systems with Output Transformation
}

\author{
Francisco González de Cossío, Madiha Nadri and Pascal Dufour
}

\begin{abstract}
This paper addresses the problem of robust observer design for nonlinear systems with transformed output. We first assume that there exists an observer design for a given nonlinear system. The structure of the system output is often crucial for estimating the system states. Therefore, we consider the problem that arises when the output is only available under a nonlinear transformation (affected by noise). This formulation is especially useful in engineering applications, where nonlinear sensors are frequent. We propose a new interconnected observer that estimates both: the unavailable output and the system states, and we establish small-gain type conditions ensuring the asymptotic convergence of the overall error dynamics. To explicit our design parameters, we study the particular cases of stateaffine systems up to output injection and systems with additive triangular nonlinearities. Finally, we provide corresponding numerical examples and comparisons with known observers.
\end{abstract}

\section{INTRODUCTION}

The problem of observer design for nonlinear systems has been a major research topic in control for many decades. Common applications include process monitoring, diagnosis and control. Indeed, it is often the case that nonlinear control laws depend on state information making the observer an essential estimation tool [1], [2]. We next discuss some of the available approaches to observer design.

The usual observers for linear systems are the so-called Luenberger observer and Kalman observer, which can be extended to nonlinear systems in specific forms. Therefore, one common strategy is to look for a coordinate transformation that steers the system into a convenient form [3], [4]. Early important contributions in this direction include: a linear form with output injection [5], a bilinear form with output injection and its Kalman-like observer [6], [7], and a triangular form for uniformly observable system and its Luenberger-like or highgain observer [8]. Indeed, a popular approach for observer design for nonlinear systems consists in compensating the Lipschitz nonlinearities by using high-gain. As stated in [9], the two early and fundamental works [8], [10] start different research directions for high-gain observers. These directions concern: (i) global results under global growth conditions, and (ii) the interactions between the peaking phenomena and the nonlinearities. Although relatively simple and fast, high-gain observers tend to amplify measurement noise for large tuning parameters [11], [12]. Hence, a useful strategy consists in adapting the gain of the observer through time, see for example [12], [13], [14].

F. González de Cossío, M. Nadri and P. Dufour are with Univ. Lyon, Université Claude Bernard Lyon 1, CNRS, LAGEPP UMR 5007, 43 boulevard du 11 novembre 1918, F-69100, Villeurbanne, France (corresponding author: madiha.nadri-wolf@univ-lyon1.fr; francisco.gonzalez-de-cossio@univlyon1.fr, pascal.dufour@univ-lyon1.fr).
Even though these observer designs mainly concern nonlinear systems, their outputs tend to be linear. Accordingly, a common goal of sensor manufacturers is achieving linearity. This is often complicated since a large number of classical sensors, especially in engineering systems, exhibit nonlinear behavior [15], [16]. For example, this is the case in: fuel cell power systems [17], image restoration [18], digital imaging [19], combustion control in automobiles [20] and engineering medicine [21].

A popular choice of an observer for general nonlinear systems with nonlinear output is the Extended Kalman Filter (EKF) in its deterministic version. Despite its simplicity and noise filtering properties, it is not always implemented since only local convergence is guaranteed [22]. The early work in [3] develops a global observer but for systems in observability canonical form with a smooth nonlinear output. On the other hand, systems with monotonic nonlinearities are studied in [23], where the authors remove the Lipschitz condition and avoid high-gain by using the so-called circle criterion. In [24], they expand these results and consider nonlinear outputs in the presence of model uncertainties. The work in [25] instead deals with a more general type of nonlinearities, those satisfying incremental quadratic constraints. Finally, the authors in [26] propose a simple observer design for systems in triangular form with a nonlinear output. This output function is not necessarily differentiable but it must satisfy an incremental sector condition.

The results above, except for the EKF, require systems in specific forms and sufficient conditions for the existence of the corresponding coordinate transformations are usually strong [3], [27], [28]. Moreover, finding the right transformations can be difficult, especially in the multi-output case. Another limitation is that measurement noise is often ignored. A natural framework for studying the robustness of an observer with respect to measurement noise is that of input-to-state stability (ISS) as introduced in [29], for a summary see [30]. Indeed, we can consider the error dynamics as the state and the measurement noise as the input [31]. In this context, ISS is referred to as disturbance-to-error stability (DES) [32], [33], [34].

In this paper, we first suppose that a DES observer has been designed for a given nonlinear system with an output $y$. This design cannot be directly implemented if we instead measure a nonlinear transformation $\psi(y)$. Therefore, we propose a new interconnected observer that estimates both: $y$ and the system states. We suppose that $\psi$ is a local diffeomorphism, however, $\psi$ can be difficult to invert or its inverse might not be available in closed form. In order to study the robustness of the new observer, we consider model uncertainties on $y$ as in [24] and 
measurement noise on $\psi(y)$. We use small-gain arguments [35] to show that the new observer is asymptotically convergent to a neighborhood of the origin that depends on the amplitude of the disturbances (or convergent to zero in their absence). Our observer design is partially inspired by the Newton-Raphson method and [36], where the authors develop an explicit observer for systems defined on a manifold given by algebraic equations. On the other hand, the authors in [37] assume the existence of a Lyapunov function for the error dynamics of an observer that cannot be directly used, given that the output is only measured through a second linear system. We apply our general results to two families of systems: state affine systems up to output injection and systems with additive triangular nonlinearity. These families differ considerably since they represent non-uniformly and uniformly observable systems respectively. We finish by providing numerical examples for both cases.

The present work improves and extends our CDC conference version [38]. In particular: (i) we give a proof of the asymptotic convergence to zero of the new observer in the case of no disturbances, (ii) we present a more adequate version of the small-gain theorem and we provide detailed proofs in the Appendix, (iii) we apply our results to a new class of systems and we make a direct comparison with the observer given in [26].

The paper is organized as follows. Section II includes the standard notation, the problem statement and adapted results from the ISS theory. Section III presents the main contribution of this paper, that is, the new observer design and the proof of its asymptotic convergence. Section IV studies the developed theory for two specific families of systems, and Section $\mathrm{V}$ provides the two corresponding numerical examples. Finally, Section VI gathers some brief concluding remarks and the Appendix presents some proofs from the ISS framework.

\section{FRAMEWORK}

\section{A. Notation}

- $\mathbb{R}^{+}$stands for the non-negative real numbers. We consider elements of $\mathbb{R}^{n}$ as column vectors.

- The transpose of the real matrix $M$ is denoted by $M^{\prime}$. If $M$ is symmetric, then $\lambda_{\min }(M)$ and $\lambda_{\max }(M)$ stand for the minimum and the maximum eigenvalue of $M$. The $n$-dimensional identity matrix is $I_{n}$.

- $|\cdot|$ denotes the spectral norm of a matrix or the Euclidean norm of a vector. That is, $|M|^{2}=\lambda_{\max }\left(M^{\prime} M\right)$.

- We denote the pre-image of 0 under $z: \mathbb{R}^{+} \rightarrow \mathbb{R}^{n}$ as $z^{-1}(0)$, that is, all $t \in \mathbb{R}^{+}$such that $z(t)=0$. As usual, $|z|_{\infty}=\sup _{t \in \mathbb{R}^{+}}|z(t)|$.

- The abbreviation "a.e." stands for "almost every" and means for all except for a measure zero subset.

\section{B. Problem statement}

Let us consider a nonlinear system of the form

$$
\left\{\begin{array}{l}
\dot{x}=f(x, u) \\
y=h(x)+d,
\end{array}\right.
$$

where $x \in \mathbb{R}^{n}$ is the state, $y \in \mathbb{R}^{p}$ the output, $u \in \mathbb{R}^{m}$ an input and $d \in \mathbb{R}^{p}$ is any locally Lipschitz disturbance. We suppose that $f$ and $h$ are of class $C^{2}$, that the system is forward-complete and we denote the input set by $u \in \mathcal{U}$.

We first assume that a "robust" observer has been designed for system (1) and that is given by

$$
\left\{\begin{array}{l}
\dot{\hat{x}}=\hat{f}(\hat{x}, g, y, u) \\
\dot{g}=G(g, u),
\end{array}\right.
$$

where $\hat{x} \in \mathbb{R}^{n}$ is the state estimation, $g$ a dynamic gain and $\hat{f}$ is locally Lipschitz.

We then consider the case where $y$ is not directly available for measurements. Instead, we measure a nonlinear transformation $\psi(y)$ affected by noise. This situation arises frequently in engineering processes, where nonlinear sensor transformations are common. The new system takes the form

$$
\left\{\begin{array}{l}
\dot{x}=f(x, u) \\
y_{\psi}=\psi(y)+d_{\psi},
\end{array}\right.
$$

where $y_{\psi} \in \mathbb{R}^{p}$ is the output, $y=h(x)+d_{y}$, the disturbances $d_{y}, d_{\psi} \in \mathbb{R}^{p}$ are bounded with bounded derivatives and $\psi$ is of class $C^{2}$. Here, $d_{y}$ can represent model uncertainties while $d_{\psi}$ measurement noise. As a consequence of changing the system output, observer (2) cannot be directly implemented and, thus, a redesign is needed.

For a general nonlinear system, there is no systematic way to adapt a given observer design to output transformations. In this work, we provide a novel method of observer redesign that faces this challenge.

Remark 1: As an example, consider the uniformly observable systems as in Section IV-B. Many observers are easily designed for this class of systems, however, they are based on a linear output. If a nonlinear transformation of the output is instead measured, the convergence of these observers is no longer guaranteed.

Remark 2: Other solutions to our problem include: (i) coordinate change to steer system (3) into a convenient form. This can be difficult and it is not systematic, especially for multi-output systems [3], [27]; (ii) using the EKF for system (3) without guarantee of its global convergence [22]; (iii) inverting $\psi$ and using observer (2). Unfortunately, the inverse of $\psi$ might not be available in closed form or it can be difficult to compute.

We cannot implement observer (2) since $y$ is not directly known. However, we require this observer to be robust with respect to measurement noise. The error dynamics are given by

$$
\dot{e}=\mathcal{F}(t, e, d)
$$

where $e=x-\hat{x}$ and for

$$
\begin{aligned}
\mathcal{F}(t, e, d)= & f(x(t), u(t)) \\
& -\hat{f}(x(t)-e, g(t), h(x(t))+d, u(t)),
\end{aligned}
$$

therefore, what we need is the following assumption. 
Assumption 1: There exists a continuous function $\bar{V}(t, e)$ : $\mathbb{R}^{+} \times \mathbb{R}^{n} \rightarrow \mathbb{R}^{+}$, of class $C^{1}$ on $e \neq 0$, and functions $\bar{\alpha}_{1}$, $\bar{\alpha}_{2} \in \mathcal{K}_{\infty}$ and $\bar{\alpha}_{3}, \bar{\chi} \in \mathcal{K}$ such that:

$$
\bar{\alpha}_{1}(|e|) \leq \bar{V}(t, e) \leq \bar{\alpha}_{2}(|e|),
$$

for all $t \in \mathbb{R}^{+}$and all $e \in \mathbb{R}^{n}$, and such that:

$$
\frac{\partial \bar{V}}{\partial t}(t, e)+\frac{\partial \bar{V}}{\partial e}(t, e) \mathcal{F}(t, e, d) \leq-\overline{\alpha_{3}}(|e|),
$$

whenever $|e| \geq \bar{\chi}(|d|)$ and for all $t \in \mathbb{R}^{+}, e \in \mathbb{R}^{n}-\{0\}$, $d \in \mathbb{R}^{p}$ and all $u \in \mathcal{U}, x(0) \in \mathbb{R}^{n}$.

Assumption 1 is equivalent to the ISS of system (4), see [39] or, for time-varying Lyapunov functions, [40]. That is, to the graceful degradation of observer (2) performance in the presence of measurement noise. Observers satisfying this property were first considered in [31] and they are known as disturbance-to-error stable (DES) observers [32]. There are methods to determine if certain observers are DES [34] or to redesign them if they are not [33].

Assumption 2: The function $\psi: \mathbb{R}^{p} \rightarrow \mathbb{R}^{p}$ is of class $C^{2}$ and its Jacobian is invertible on all its domain. Moreover, there exists $\delta \in \mathcal{K}_{\infty} \cap C^{2}$ such that

$$
\delta(|\psi(y)-\psi(\hat{y})|) \geq|y-\hat{y}|,
$$

for all $y, \hat{y} \in \mathbb{R}^{p}$.

Assumption 2 implies in particular the injectivity of $\psi$. It is satisfied, for example, if $p=1$ and if $\left|\frac{\partial \psi}{\partial y}\right|$ is bounded from below by a positive constant. The authors in [26] require this last condition to hold when the nonlinear output they study is differentiable.

\section{Input to state practical stability}

The ISS theory has been the subject of intense research since its introduction in [29]. It relates the states of a given system with the initial conditions and the inputs through the so-called comparison functions: (i) $\gamma: \mathbb{R}^{+} \rightarrow \mathbb{R}^{+}$is of class $\mathcal{K}$ if it is strictly increasing, continuous and $\gamma(0)=0$. If additionally $\gamma(t)$ tends to infinity as $t \rightarrow \infty$, then it is of class $\mathcal{K}_{\infty}$, (ii) $\beta: \mathbb{R}^{+} \times \mathbb{R}^{+} \rightarrow \mathbb{R}^{+}$is of class $\mathcal{K} \mathcal{L}$ if for each fixed $t \in \mathbb{R}^{+}$ the function $\beta(s, t)$ is of class $\mathcal{K}$ and if for each fixed $s \in \mathbb{R}^{+}$ the function $\beta(s, t)$ is decreasing and tends to zero as $t \rightarrow \infty$. We use the notation $\gamma \in \mathcal{K}$ or $\gamma \in \mathcal{K}_{\infty}$ and $\beta \in \mathcal{K} \mathcal{L}$. A comprehensive study of the function classes $\mathcal{K}$ and $\mathcal{K} \mathcal{L}$ can be found in [41]. The following general results will be used in Section III.

Remark 3: The function classes $\mathcal{K}$ and $\mathcal{K}_{\infty}$ are closed under composition. Also, functions in $\mathcal{K}_{\infty}$ are invertible and their inverses remain in the class. A frequently used triangletype inequality for $\gamma \in \mathcal{K}$ is

$$
\gamma(s+t) \leq \gamma(2 s)+\gamma(2 t)
$$

for all $s, t \in \mathbb{R}^{+}$.

Proposition 1 ([42]): For any locally Lipschitz function $\phi: \mathbb{R}^{n_{1}} \rightarrow \mathbb{R}^{n_{2}}$, there exist locally Lipschitz functions $\varphi$ : $\mathbb{R}^{n_{1}} \rightarrow \mathbb{R}^{+}$and $\alpha \in \mathcal{K}_{\infty}$ such that

$$
|\phi(z)-\phi(w)| \leq \varphi(z) \alpha(|z-w|),
$$

for all $z, w \in \mathbb{R}^{n_{1}}$.

A weaker notion than ISS is the so-called input-to-state practical stability (ISpS) as defined in [43]. Here, we write the definition for a family of function pairs. As can be seen in the Appendix, this does not modify the usual results concerning ISpS and it simplifies our exposition.

Definition 1: Consider any family $\mathcal{G}$ formed by pairs of locally Lipschitz functions $(z, w): I_{z, w}=\left[0, T_{z, w}\right) \rightarrow \mathbb{R}^{n_{1}} \times$ $\mathbb{R}^{n_{2}}$. We say that $\mathcal{G}$ is practically stable if there exist functions $\beta \in \mathcal{K} \mathcal{L}$ and $\gamma \in \mathcal{K}$ and a constant $c \geq 0$ such that for all $(z, w) \in \mathcal{G}$ we have

$$
|z(t)| \leq \beta(|z(0)|, t)+\sup _{s \in[0, t]} \gamma(|w(s)|)+c, \quad \forall t \in I_{z, w} .
$$

The functions $\beta$ and $\gamma$ and the constant $c$ are respectively called decay rate, gain and constant of the practical stability.

The "practicality" comes from the constant $c$ appearing in the right-hand side of equation (6). In fact, if $c=0$ then we recover the ISS property. It is known that the practical stability of a system can be proved by constructing a Lyapunov function [44]. In order to enlarge the class of admissible Lyapunov functions, the next definition uses the fact that locally Lipschitz functions are differentiable almost everywhere (Rademacher's theorem).

Definition 2: A function $V: \mathbb{R}^{+} \times \mathbb{R}^{n_{1}} \rightarrow \mathbb{R}^{+}$is a Lyapunov function for the family $\mathcal{G}$ if: (i) for each $(z, w) \in \mathcal{G}$, $V^{z}=V(\cdot, z(\cdot))$ is everywhere continuous and locally Lipschitz outside $z^{-1}(0)$, (ii) there exist $\alpha_{1}, \alpha_{2} \in \mathcal{K}_{\infty}, \alpha_{3}, \chi \in \mathcal{K}$ and $c_{L} \geq 0$ such that for all $(z, w) \in \mathcal{G}$ :

$$
\alpha_{1}(|z(t)|) \leq V^{z}(t) \leq \alpha_{2}(|z(t)|),
$$

for all $t \in I_{z, w}$ and

$$
\dot{V}^{z}(t) \leq-\alpha_{3}(|z(t)|),
$$

if $|z(t)| \geq \chi(|w(t)|)+c_{L}$ and for a.e. $t \in I_{z, w}-z^{-1}(0)$. The functions $\alpha_{i}$, the function $\chi$ and the constant $c_{L}$ are respectively called Lyapunov-bounds, gain and constant.

Theorem 1 (Lyapunov function): Consider any family $\mathcal{G}$ formed by pairs of locally Lipschitz functions. Then $\mathcal{G}$ is practically stable if it has a Lyapunov function.

The proof of Theorem 1 goes along the lines of that in [39] and it is included in the Appendix for completeness. We remark that $c=0$ precisely when $c_{L}=0$.

The stability of the interconnection of ISpS subsystems can be achieved by imposing a small-gain condition as in [43], [35] and, for large networks, [45]. In our setting, let us define the set

$$
\mathcal{G}^{-1}=\{(w, z) \mid(z, w) \in \mathcal{G}\}
$$

and suppose that:

- there is a Lyapunov function for $\mathcal{G}$ with corresponding Lyapunov-bounds, gain and constant: $\alpha_{11}, \alpha_{12}$ and $\alpha_{13}$, $\chi_{1}$ and $c_{L 1}$,

- there is a Lyapunov function for $\mathcal{G}^{-1}$ with corresponding Lyapunov-bounds, gain and constant: $\alpha_{21}, \alpha_{22}$ and $\alpha_{23}$, $\chi_{2}$ and $c_{L 2}$. 


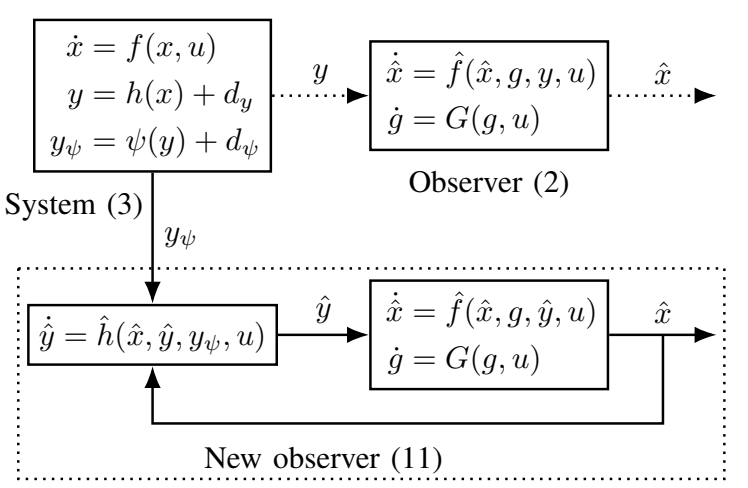

Figure 1. System (3) and observer (2) are represented on the top part of the diagram. The new observer (11) is represented on the bottom part as an interconnected system. Observer (2) requires the unavailable output $y$, while the new observer uses the measurements $y_{\psi}$ instead.

We can then define the mixed Lyapunov gains as:

$$
\chi_{m 1}=\alpha_{12}\left(2 \chi_{1}\left(\alpha_{21}^{-1}\right)\right), \quad \chi_{m 2}=\alpha_{22}\left(2 \chi_{2}\left(\alpha_{11}^{-1}\right)\right)
$$

and we have the following result.

Theorem 2 (small-gain): Consider any family $\mathcal{G}$ formed by pairs of locally Lipschitz functions $(z, w): I_{z, w}=$ $\left[0, T_{z, w}\right) \rightarrow \mathbb{R}^{n_{1}} \times \mathbb{R}^{n_{2}}$. Suppose that there exist a Lyapunov function for $\mathcal{G}$ and a Lyapunov function for $\mathcal{G}^{-1}$. Given the mixed Lyapunov gains in (9), if $\chi_{1}$ is of class $\mathcal{K}_{\infty}$ and if

$$
\chi_{m 1}\left(\chi_{m 2}(t)\right)<t, \quad \forall t>0
$$

then $\mathcal{G} \times\{0\}$ is practically stable. That is, there exist a function $\beta \in \mathcal{K} \mathcal{L}$ and a constant $c \geq 0$ such that for all $(z, w) \in \mathcal{G}$ we have

$$
|(z(t), w(t))| \leq \beta(|(z(0), w(0))|, t)+c, \quad \forall t \in I_{z, w} .
$$

The proof of Theorem 2, given in Appendix B, is based on the proof of the small-gain theorem in [35]. As before, $c=0$ precisely when $c_{L 1}=c_{L 2}=0$.

\section{NEW OBSERVER DESIGN}

We propose the observer design for system (3) as the following interconnection:

$$
\left\{\begin{array}{l}
\dot{\hat{x}}=\hat{f}(\hat{x}, g, \hat{y}, u) \\
\dot{g}=G(g, u) \\
\dot{\hat{y}}=\hat{h}\left(\hat{x}, \hat{y}, y_{\psi}, u\right),
\end{array}\right.
$$

where $\hat{f}$ and $G$ are as in (2),

$$
\begin{aligned}
\hat{h}\left(\hat{x}, \hat{y}, y_{\psi}, u\right)= & \frac{\partial \psi}{\partial y}(\hat{y})^{-1} \frac{\partial \psi}{\partial y}(h(\hat{x})) \frac{\partial h}{\partial x}(\hat{x}) f(\hat{x}, u) \\
& +\frac{\partial \psi}{\partial y}(\hat{y})^{-1} \varphi(\hat{x}, u) K\left(y_{\psi}-\psi(\hat{y})\right),
\end{aligned}
$$

and $\varphi: \mathbb{R}^{n} \times \mathbb{R}^{m} \rightarrow \mathbb{R}^{+}$and $K: \mathbb{R}^{p} \rightarrow \mathbb{R}^{p}$ are locally Lipschitz functions defined next. We emphasize that the observer in (11) only requires knowledge of $y_{\psi}$ and not directly of $y$. Figure 1 compares observers (2) and (11) and illustrates the relation of these observers with system (3).
In order to define the function $\varphi$, let us first consider the function $\phi$ given by

$$
\begin{aligned}
& \phi\left(x, u, d_{1}, d_{2}, d_{3}\right)= \\
& \quad \frac{\partial \psi}{\partial y}\left(h(x)+d_{1}\right)\left(\frac{\partial h}{\partial x}(x) f(x, u)+d_{2}\right)+d_{3},
\end{aligned}
$$

for all $x \in \mathbb{R}^{n}, u \in \mathbb{R}^{m}$ and $d_{1}, d_{2}, d_{3} \in \mathbb{R}^{p}$. According to Proposition 1, there exist locally Lipschitz functions $\varphi$ : $\mathbb{R}^{n} \times \mathbb{R}^{m} \rightarrow \mathbb{R}^{+}$and $\alpha \in \mathcal{K}_{\infty}$ such that,

$$
\begin{aligned}
\mid \phi\left(x, u, d_{1}, d_{2}, d_{3}\right)- & \phi(\hat{x}, u, 0,0,0) \mid \leq \\
& \varphi(\hat{x}, u) \alpha\left(\left|\left(x-\hat{x}, d_{1}, d_{2}, d_{3}\right)\right|\right)
\end{aligned}
$$

on all its domain and we can assume that $\varphi \geq 1$.

Remark 4: Finding $\alpha$ and $\varphi$ in (13) is simpler if $\psi$ and the functions in expression (12) satisfy global Lipschitz conditions. This is the case, for example, if system (3) has bounded states and if we use saturation techniques as explained in Remark 7. We can then take $\alpha$ as the identity function and

$$
\varphi(\hat{x}, u) \geq c_{\varphi}(|f(\hat{x}, u)|+1),
$$

for some $c_{\varphi} \geq 1$ and for all $\hat{x} \in \mathbb{R}^{n}$ and all $u \in \mathbb{R}^{m}$. There is a vast literature dealing with the computation of Lipschitz constants like those defining $c_{\varphi}$.

We now continue with the definition of $K$. Consider a function $\rho \in \mathcal{K}_{\infty} \cap C^{2}$, a positive constant $k$ and set $K: \mathbb{R}^{p} \rightarrow \mathbb{R}^{p}$ as

$$
K(\xi)=\left\{\begin{array}{l}
\left(k \frac{\rho(|\xi|)}{|\xi|}\right) \xi, \text { if } \xi \neq 0 \\
0, \text { if } \xi=0 .
\end{array}\right.
$$

The choice of $\rho$ guarantees that $K$ is locally Lipschitz. Indeed, using that $\rho(0)=0$ and L'Hôpital's rule, we can show that the function

$$
\left\{\begin{array}{l}
\frac{\rho(t)}{t}, \text { if } t>0 \\
\dot{\rho}(0), \text { if } t=0
\end{array}\right.
$$

is continuously differentiable on $\mathbb{R}^{+}$. The function $\rho$ provides a degree of freedom for the design of the new observer (11).

Given initial conditions $\hat{x}(0) \in \mathbb{R}^{n}, \hat{y}(0) \in \mathbb{R}^{p}$ and an input $u \in \mathcal{U}$, there is a corresponding maximal interval of existence $[0, T)$ for the unique solution $(\hat{x}, \hat{y})$ of $(11)$. We denote the estimation errors as

$$
e(t)=x(t)-\hat{x}(t), \quad \xi(t)=y_{\psi}(t)-\psi(\hat{y}(t)),
$$

for all $t \in[0, T)$. Notice that the solution is unbounded if $T$ is finite, see for example [46]. We will see that this is not the case if $\rho$ is properly chosen.

Lemma 1: Let Assumption 2 hold and consider systems (3) and (11) with $\varphi$ and $K$ as in (13) and (14). If $k>1$, then there exists a Lyapunov function for the family

$$
\mathcal{G}=\left\{(\xi, e) \mid x(0), \hat{x}(0) \in \mathbb{R}^{n}, \hat{y}(0) \in \mathbb{R}^{p}, u \in \mathcal{U}\right\},
$$

where $\xi=y_{\psi}-\psi(\hat{y})$ and $e=x-\hat{x}$ are defined on $[0, T)$. Moreover, if the disturbances $d_{y}$ and $d_{\psi}$ are both zero then the corresponding Lyapunov-constant can be chosen to be zero. 
Proof: We propose the Lyapunov function simply as the norm. On the trajectory $\xi$, it takes the form

$$
V_{1}^{\xi}(t)=|\xi(t)|
$$

for all $t \in[0, T)$. It is clear that property (7) is satisfied by defining both $\alpha_{11}$ and $\alpha_{12}$ as the identity function.

We next prove that property (8) is also satisfied on $[0, T)-$ $\xi^{-1}(0)$. From the definitions of $\phi$ in (12) and of the new observer in (11) we have that

$$
\begin{aligned}
\dot{V_{1}^{\xi}}= & \frac{\xi^{\prime}}{|\xi|}\left(\frac{\partial \psi}{\partial y}(y) \dot{y}+\dot{d}_{\psi}-\frac{\partial \psi}{\partial y}(\hat{y}) \dot{\hat{y}}\right) \\
= & \frac{\xi^{\prime}}{|\xi|}\left(\phi\left(e+\hat{x}, u, d_{y}, \dot{d}_{y}, \dot{d}_{\psi}\right)-\phi(\hat{x}, u, 0,0,0)\right) \\
& -\frac{\xi^{\prime}}{|\xi|} \varphi(\hat{x}, u) K(\xi)
\end{aligned}
$$

where $\xi^{\prime}$ denotes the transpose of $\xi$. It then follows from the construction of $\varphi$ and $K$, respectively in (13) and (14), and by Remark 3 that

$$
\begin{aligned}
\dot{V}_{1}^{\xi} & \leq \varphi(\hat{x}, u) \alpha\left(\left|\left(e, d_{y}, \dot{d}_{y}, \dot{d}_{\psi}\right)\right|\right)-k \varphi(\hat{x}, u) \rho(|\xi|) \\
& \leq \varphi(\hat{x}, u)\left(\alpha(2|e|)+\alpha\left(c_{d}\right)-k \rho(|\xi|)\right)
\end{aligned}
$$

where

$$
c_{d}=2\left(\left|d_{y}\right|_{\infty}+\left|\dot{d}_{y}\right|_{\infty}+\left|\dot{d_{\psi}}\right|_{\infty}\right) .
$$

On the other hand, suppose that $\chi_{1} \in \mathcal{K}$ is given by

$$
\chi_{1}(t)=\rho^{-1}(2 \alpha(2 t)),
$$

for all $t \in \mathbb{R}^{+}$, and set the non-negative constant

$$
c_{L 1}=\rho^{-1}\left(2 \alpha\left(c_{d}\right)\right) \text {. }
$$

If $|\xi| \geq \chi_{1}(|e|)+c_{L 1}$, then it follows that

$$
\rho(|\xi|) \geq \alpha(2|e|)+\alpha\left(c_{d}\right) .
$$

Since $k>1$ and $\varphi \geq 1$, we get that the negative term $\rho(|\xi|)-$ $k \rho(|\xi|)$ dominates the last expression in (16). By using this and the inequalities in (16) we conclude that

$$
\dot{V_{1}^{\xi}} \leq-(k-1) \rho(|\xi|)
$$

and, as a consequence, we can define

$$
\alpha_{13}(t)=(k-1) \rho(t),
$$

for all $t \in \mathbb{R}^{+}$. Finally, notice that if both disturbances $d_{y}$ and $d_{\psi}$ are the zero function then $c_{d}$ in (17) is zero and the same holds for the Lyapunov-constant $c_{L 1}$.

Lemma 2: Let Assumptions 1 and 2 hold and consider systems (3) and (11) with $\varphi$ and $K$ as in (13) and (14). There exists a Lyapunov function for the family

$$
\mathcal{G}^{-1}=\left\{(e, \xi) \mid x(0), \hat{x}(0) \in \mathbb{R}^{n}, \hat{y}(0) \in \mathbb{R}^{p}, u \in \mathcal{U}\right\},
$$

where $e=x-\hat{x}$ and $\xi=y_{\psi}-\psi(\hat{y})$ are defined on $[0, T)$. Moreover, if the disturbances $d_{y}$ and $d_{\psi}$ are both zero then the corresponding Lyapunov-constant can be chosen to be zero.

Proof: Consider the Lyapunov function $\bar{V}$ from Assumption 1 and the corresponding functions $\bar{\alpha}_{1}, \bar{\alpha}_{2}, \bar{\alpha}_{3}$ and $\bar{\chi}$. Set $V_{2}=\bar{V}$, which on the trajectory takes the form $V_{2}^{e}=V_{2}(\cdot, e)$, and define $\alpha_{21}=\bar{\alpha}_{1}$ and $\alpha_{22}=\bar{\alpha}_{2}$. We next prove that property (8) holds on $[0, T)-e^{-1}(0)$. From the definition of $\mathcal{F}$ in (5) we have that

$$
\dot{e}=\mathcal{F}(\cdot, e, \hat{y}-h(x))
$$

and, therefore, we can select $\alpha_{23}=\bar{\alpha}_{3}$ to get

$$
\begin{aligned}
\dot{V}_{2}^{e} & =\frac{\partial \bar{V}}{\partial t}(\cdot, e)+\frac{\partial \bar{V}}{\partial z}(\cdot, e) \dot{e} \\
& \leq-\alpha_{23}(|e|),
\end{aligned}
$$

whenever $|e| \geq \bar{\chi}(|\hat{y}-h(x)|)$. We also define the class $\mathcal{K}$ function

$$
\chi_{2}(t)=\bar{\chi}(2 \delta(2 t)),
$$

for all $t \in \mathbb{R}^{+}$, and the non-negative constant

$$
c_{L 2}=\bar{\chi}\left(2\left|d_{y}\right|_{\infty}\right)+\bar{\chi}\left(2 \delta\left(2\left|d_{\psi}\right|_{\infty}\right)\right) .
$$

Assumption 2 then implies that

$$
\begin{aligned}
\chi_{2}(|\xi|)+c_{L 2} & \geq \bar{\chi}(2 \delta(|\psi(y)-\psi(\hat{y})|))+\bar{\chi}\left(2\left|d_{y}\right|_{\infty}\right) \\
& \geq \bar{\chi}(2|y-\hat{y}|)+\bar{\chi}\left(2\left|d_{y}\right|_{\infty}\right) \\
& \geq \bar{\chi}\left(|y-\hat{y}|+\left|d_{y}\right|_{\infty}\right) \\
& \geq \bar{\chi}(|\hat{y}-h(x)|)
\end{aligned}
$$

which, together with (20), provides the needed property. Finally, notice that $c_{L 2}$ is zero precisely when the disturbances $d_{y}$ and $d_{\psi}$ are zero.

We are now ready to state our main result. It establishes a condition on the gain $\rho$ in order to guarantee the asymptotic convergence to a neighborhood of zero (or to zero itself) of the state estimation error given by the new observer in (11).

Theorem 3 (Observer gain design): Let Assumptions 1 and 2 hold and consider systems (3) and (11) with $\varphi$ and $K$ as in (13) and (14). For any $k>1$ and any $\rho \in \mathcal{K}_{\infty} \cap C^{2}$ satisfying

$$
\rho(t)>2 \alpha\left(2 \bar{\alpha}_{1}^{-1}\left(\bar{\alpha}_{2}(2 \bar{\chi}(2 \delta(4 t)))\right)\right), \quad \forall t>0
$$

there exist a class $\mathcal{K} \mathcal{L}$ function $\beta$ and a constant $c \geq 0$ such that for all $x(0), \hat{x}(0) \in \mathbb{R}^{n}$, all $\hat{y}(0) \in \mathbb{R}^{p}$ and all $u \in \mathcal{U}$ the estimation errors $e=x-\hat{x}$ and $\xi=y_{\psi}-\psi(\hat{y})$ are defined on $\mathrm{R}^{+}$and

$$
|(\xi(t), e(t))| \leq \beta(|(\xi(0), e(0))|, t)+c, \quad \forall t \geq 0 .
$$

Moreover, if the disturbances $d_{y}$ and $d_{\psi}$ are both zero then $c$ is zero as well.

Proof: Using Lemmas 1 and 2, we deduce that there exist Lyapunov functions for the family $\mathcal{G}$ given in (15) and for the family $\mathcal{G}^{-1}$ given in (19). Since the first two Lyapunov-bounds in the proof of Lemma 1 can be taken as the identity function, the small-gain condition in (10) is given by:

$$
\chi_{m 1}=2 \chi_{1}\left(\alpha_{21}^{-1}\right), \quad \chi_{m 2}=\alpha_{22}\left(2 \chi_{2}\right) .
$$

It is then straight-forward to verify the equivalence of this condition with the inequality in (21). Indeed, by using the expression of $\chi_{1}$ in (18) and the functions defined in the proof of Lemma 2 we have that

$$
2 \chi_{1}\left(\alpha_{21}^{-1}\left(\alpha_{22}\left(2 \chi_{2}(t)\right)\right)\right)<t, \quad \forall t>0
$$


precisely when $\rho$ satisfies (21). Hence, Theorem 2 concludes that $\mathcal{G} \times\{0\}$ is practically stable. That is, there exist a function $\beta \in \mathcal{K} \mathcal{L}$ and a constant $c \geq 0$ such that (22) is satisfied on $[0, T)$. As a consequence, this interval is necessarily the whole $\mathbb{R}^{+}$.

Finally, if both disturbances $d_{y}$ and $d_{\psi}$ are zero then in Lemmas 1 and 2 both Lyapunov-constants $c_{L 1}$ and $c_{L 2}$ are zero as well. The details of the proofs in the Appendix imply then $c=0$.

Remark 5: In practice, the design of the new observer (11) starts by proposing an observer as (2) and by finding a Lyapunov function $\bar{V}$ for its error dynamics, together with the functions $\bar{\alpha}_{1}, \bar{\alpha}_{2}$ and $\bar{\chi}$ from Assumption 1. We then require $\delta$ from Assumption 2 and the functions $\varphi$ and $\alpha$ from (13) (see Remark 4). Finally, the lower bound in (21) itself can be used to construct such a $\rho$ and $K$ is then given by (14).

Remark 6: From the proofs of Lemmas 1 and 2, we have that the new observer recovers a type of DES property: there exist $\beta \in \mathcal{K} \mathcal{L}$ and $\gamma \in \mathcal{K}$ such that for all $x(0), \hat{x}(0) \in \mathbb{R}^{n}$, all $\hat{y}(0) \in \mathbb{R}^{p}$, all $u \in \mathcal{U}$ and for all bounded, Lipschitz and differentiable $d_{y}$ and $d_{\psi}$ we have

$$
|(\xi(t), e(t))| \leq \beta(|(\xi(0), e(0))|, t)+\gamma\left(\left|\left(d_{y}, d_{\psi}, \dot{d}_{y}, \dot{d}_{\psi}\right)\right|_{\infty}\right),
$$

for all $t \in \mathbb{R}^{+}$.

Finally, notice that we can find the explicit decay rate $\beta$ and constant $c$ in (22) by following the next steps:

1) Find the Lyapunov-bounds, gain and constant from Assumption 2. Use them to construct $\rho$ satisfying the inequality in (21).

2) Get the Lyapunov-bounds, gains and constants from Lemma 1 and Lemma 2.

3) Consider the mixed gains in (23) and choose an inbetween function $\sigma$ as explained in the Appendix.

4) Compute the bounds, gain and constant of the Lyapunov function for $\mathcal{G} \times\{0\}$ as in the proof of Theorem 2 in the Appendix.

5) Compute the corresponding decay rate $\beta$ and constant $c$ as shown in the proofs of Lemma 3 and Theorem 1 in the Appendix.

The following section concerns two classical and widely used families of nonlinear systems and it helps to illustrate the design of the new observer.

\section{PARTICUlar CASES}

The aim of this section is to study Theorem 3 when system (1) belongs to the family of: (i) state affine systems, (ii) systems with additive triangular nonlinearity. We add the following assumption on $\psi$ throughout this section.

Assumption 3: The function $\psi$ and its derivative are both Lipschitz continuous.

This assumption is not a critical design requirement but it simplifies the computations of $\varphi$ and $\alpha$ in (13) as explained in Remark 4. Moreover, it can be easily met in the case of bounded states as shown in Remark 7 at the end of this section.

\section{A. State-affine systems up to output injection}

Consider $A: \mathbb{R}^{m} \rightarrow \mathbb{R}^{n \times n}$ a continuous matrix functional, $\eta: \mathbb{R}^{p} \times \mathbb{R}^{m} \rightarrow \mathbb{R}^{n}$ a nonlinear and continuous function and $C \in \mathbb{R}^{p \times n}$ a constant matrix. Our goal is to estimate the states of the system given by:

$$
\left\{\begin{array}{l}
\dot{x}=A(u) x+\eta(C x, u) \\
y_{\psi}=\psi(y)+d_{\psi},
\end{array}\right.
$$

where $y=C x+d_{y}$ and where $\psi: \mathbb{R}^{p} \rightarrow \mathbb{R}^{p}$ satisfies Assumption 2. We need the next condition on $\eta$.

Assumption 4: The function $\eta$ is Lipschitz continuous with respect to its first entrance. That is, there exists a positive constant $c_{\eta}$ such that

$$
|\eta(y, u)-\eta(\hat{y}, u)| \leq c_{\eta}|y-\hat{y}|,
$$

for all $y, \hat{y} \in \mathbb{R}^{p}$ and all $u \in \mathbb{R}^{m}$.

The following observer [7], [47], which cannot be directly implemented, plays the role of observer (2) and it is described by:

$$
\left\{\begin{array}{l}
\dot{\hat{x}}=A(u) \hat{x}+\eta(y, u)+S^{-1} C^{\prime}(y-C \hat{x}) \\
\dot{S}=-\theta S-A(u)^{\prime} S-S A(u)+C^{\prime} C,
\end{array}\right.
$$

where $\theta>0$ is a tuning parameter and $S(0) \in \mathbb{R}^{n \times n}$ is a symmetric and positive definite matrix. It is known that $S(t)$ maintains these properties for all $t \in \mathbb{R}^{+}$.

The equation defining $S$ in (25) is the same as in [48]. Following their work, the input $u$ is regularly persistent if there exist $a>0, T>0$ and $t_{0} \geq T$ such that

$$
\int_{t-T}^{t} \Phi_{u}(s, t-T)^{\prime} C^{\prime} C \Phi_{u}(s, t-T) d s \geq a I_{n},
$$

for all $t \geq t_{0}$ and where $\Phi_{u}$ stands for the transition matrix, namely, the unique solution to

$$
\left\{\begin{array}{l}
\frac{\partial \Phi_{u}}{\partial s}(s, t)=A(u(s)) \Phi_{u}(s, t) \\
\Phi_{u}(t, t)=I_{n},
\end{array}\right.
$$

for all $s, t \in \mathbb{R}^{+}$.

Assumption 5: The input set $\mathcal{U}$ consists of continuous, uniformly bounded and regularly persistent inputs $u: \mathbb{R}^{+} \rightarrow$ $\mathbb{R}^{m}$.

Let us consider $0<a \leq \lambda_{\min }(S(0))$ to simplify and let us denote

$$
b_{1}=\sup _{|u| \leq c_{\mathcal{U}}}|A(u)|,
$$

where $|u|_{\infty} \leq c_{\mathcal{U}}$, for all $u \in \mathcal{U}$. If $\theta \geq 3 b_{1}>0$ we can deduce the bounds [48]:

$$
s_{1} I_{n} \leq S(t) \leq s_{2} I_{n},
$$

for all $t \in \mathbb{R}^{+}$and where

$$
s_{1}=a \exp \left(-t_{0}\left(\theta+2 b_{1}\right)\right), \quad s_{2}=b_{1}^{-1}|C|^{2}+|S(0)|
$$

are positive constants.

We next show that observer (25) satisfies Assumption 1. We define the function $\bar{V}: \mathbb{R}^{+} \times \mathbb{R}^{n} \rightarrow \mathbb{R}^{+}$as

$$
\bar{V}(t, e)=e^{\prime} S(t) e,
$$


for all $t \in \mathbb{R}^{+}$and $e \in \mathbb{R}^{n}$. According to (26), we can choose the class $\mathcal{K}_{\infty}$ functions:

$$
\bar{\alpha}_{1}(t)=s_{1} t^{2}, \quad \bar{\alpha}_{2}(t)=s_{2} t^{2},
$$

for all $t \in \mathbb{R}^{+}$. Moreover, the following holds for all $t \in \mathbb{R}^{+}$, $e \in \mathbb{R}^{n}$ and $d \in \mathbb{R}^{p}$. On one hand, the definition of $S$ in (25) provides

$$
\begin{aligned}
\frac{\partial \bar{V}}{\partial t}(t, e)= & -\theta e^{\prime} S(t) e-e^{\prime} A(u(t))^{\prime} S(t) e \\
& -e^{\prime} S(t) A(u(t)) e+e^{\prime} C^{\prime} C e \\
= & -\theta e^{\prime} S(t) e-2 e^{\prime} S(t) A(u(t)) e+e^{\prime} C^{\prime} C e,
\end{aligned}
$$

where we used that $e^{\prime} A(u(t))^{\prime} S e$, as a real number, coincides with its transpose. On the other hand, $\mathcal{F}$ from (5) takes the form

$$
\begin{aligned}
\mathcal{F}(t, e, d)= & A(u(t)) e-S(t)^{-1} C^{\prime}(C e+d) \\
& +\eta(C x(t), u(t))-\eta(C x(t)+d, u(t)) .
\end{aligned}
$$

It then follows that

$$
\begin{aligned}
& \frac{\partial \bar{V}}{\partial e}(t, e) \mathcal{F}(t, e, d)=2 e^{\prime} S(t) \mathcal{F}(t, e, d) \\
& =2 e^{\prime} S(t) A(u(t)) e-2 e^{\prime} C^{\prime}(C e+d) \\
& +2 e^{\prime} S(t)(\eta(C x(t), u(t))-\eta(C x(t)+d, u(t))) .
\end{aligned}
$$

Putting (28) and (29) together we obtain that

$$
\begin{aligned}
\frac{\partial \bar{V}}{\partial t}(t, e) & +\frac{\partial \bar{V}}{\partial e}(t, e) \mathcal{F}(t, e, d)= \\
& -\theta e^{\prime} S(t) e+e^{\prime} C^{\prime} C e-2 e^{\prime} C^{\prime}(C e+d) \\
& +2 e^{\prime} S(t)(\eta(C x(t), u(t))-\eta(C x(t)+d, u(t))),
\end{aligned}
$$

and using Assumption 4 and the bounds in (26), we get

$$
\begin{aligned}
& \frac{\partial \bar{V}}{\partial t}(t, e)+\frac{\partial \bar{V}}{\partial e}(t, e) \mathcal{F}(t, e, d) \leq \\
& \quad-\theta e^{\prime} S(t) e+2|C||e||d|+2 c_{\eta}|S(t)||e||d| \leq \\
& \quad-\theta s_{1}|e|^{2}+2|C||e||d|+2 c_{\eta} s_{2}|e \| d| .
\end{aligned}
$$

We now define the class $\mathcal{K}_{\infty}$ functions

$$
\bar{\chi}(t)=s_{1}^{-1} t, \quad \bar{\alpha}_{3}(t)=s_{1}\left(\theta-b_{2}\right) t^{2},
$$

where

$$
\theta>b_{2}=2\left(|C|+c_{\eta} s_{2}\right) .
$$

Hence, the inequality $|e| \geq \bar{\chi}(|d|)$ implies that

$$
\begin{aligned}
\frac{\partial \bar{V}}{\partial t}(t, e)+\frac{\partial \bar{V}}{\partial e}(t, e) \mathcal{F}(t, e, d) & \leq s_{1}\left(-\theta+2|C|+2 c_{\eta} s_{2}\right)|e|^{2} \\
& =-\bar{\alpha}_{3}(|e|)
\end{aligned}
$$

and we are required to tune $\theta>\max \left\{3 b_{1}, b_{2}\right\}$, which is possible since $b_{1}$ and $b_{2}$ are independent of $\theta$.

We can now construct the corresponding new observer (11). Notice that in this case, the function $f$ is globally Lipschitz in $x$, uniformly in $u$ such that $|u| \leq c_{\mathcal{U}}$. Therefore, we are in the situation of Remark 4 and we can choose $\alpha$ as the identity function and

$$
\varphi(\hat{x}, u) \geq c_{\varphi}(|A(u) \hat{x}+\eta(C \hat{x}, u)|+1),
$$

for some $c_{\varphi} \geq 1$ and for all $\hat{x} \in \mathbb{R}^{n}$ and $|u| \leq c_{\mathcal{U}}$. By using the functions in (27) and (30) and by using the small-gain condition in (21), we get

$$
\rho(t)=17\left(s_{1}^{-3} s_{2}\right)^{\frac{1}{2}} \delta(4 t),
$$

for all $t \in \mathbb{R}^{+}$and where $\delta$ is given in Assumption 2. As a consequence, $K$ in (14) is given by:

$$
K(\xi)=\left\{\begin{array}{l}
17 k\left(s_{1}^{-3} s_{2}\right)^{\frac{1}{2}} \delta(4|\xi|)|\xi|^{-1} \cdot \xi, \text { if } \xi \neq 0 \\
0, \text { if } \xi=0,
\end{array}\right.
$$

for $k>1$. The observer in (11) takes the form:

$$
\left\{\begin{aligned}
\dot{\hat{x}}= & A(u) \hat{x}+\eta(\hat{y}, u)+S^{-1} C^{\prime}(\hat{y}-C \hat{x}) \\
\dot{\hat{y}}= & \frac{\partial \psi}{\partial y}(\hat{y})^{-1} \frac{\partial \psi}{\partial y}(C \hat{x}) C(A(u) \hat{x}+\eta(C \hat{x}, u)) \\
& +\frac{\partial \psi}{\partial y}(\hat{y})^{-1} \varphi(\hat{x}, u) K\left(y_{\psi}-\psi(\hat{y})\right),
\end{aligned}\right.
$$

where $S$ is as in (25). We summarize our results in the following corollary.

Corollary 1 (state-affine case): Let Assumptions 2, 3, 4 and 5 hold and consider systems (24) and (34). For any tuning parameter $\theta$ such that

$$
\theta>\max \left\{3 b_{1}, 2|C|+2 c_{\eta}\left(b_{1}^{-1}|C|^{2}+|S(0)|\right)\right\},
$$

where $b_{1}=\sup _{|u| \leq c_{\mathcal{U}}}|A(u)|$, there exist a function $\beta \in \mathcal{K} \mathcal{L}$ and a constant $c \geq 0$ such that for all $x(0), \hat{x}(0) \in \mathbb{R}^{n}$, all $\hat{y}(0) \in \mathbb{R}^{p}$ and all $u \in \mathcal{U}$, the estimation errors $e=x-\hat{x}$ and $\xi=y_{\psi}-\psi(\hat{y})$ are defined on $\mathbb{R}^{+}$and

$$
|(\xi(t), e(t))| \leq \beta(|(\xi(0), e(0))|, t)+c, \quad \forall t \geq 0 .
$$

Moreover, if the disturbances $d_{y}$ and $d_{\psi}$ are both zero then $c$ is zero as well.

Proof: It follows from our previous development and from Theorem 3. Indeed, we showed that Assumption 1 is satisfied by using Assumptions 4 and 5 .

Finally, we compute the explicit decay rate $\beta$ and the constant $c$ in (35) as explained at the end of Section III. The function $\rho$ is already given in (32), this is Step 1). For steps 2 ) and 3), we suppose that $\delta$ is linear with slope $c_{\delta}>0$ so that:

$$
\delta(t)=c_{\delta} t, \quad \rho(t)=c_{\rho} t, \quad c_{\rho}=68\left(s_{1}^{-3} s_{2}\right)^{\frac{1}{2}} c_{\delta},
$$

for all $t \in \mathbb{R}^{+}$. The mixed Lyapunov gains then take the form:

$$
\begin{array}{ll}
\chi_{m 1}(t)=c_{\chi_{m 1}} t^{\frac{1}{2}}, & c_{\chi_{m 1}}=2 s_{1}^{-\frac{1}{2}} c_{\rho}^{-1}, \\
\chi_{m 2}(t)=c_{\chi_{m 2}} t^{2}, & c_{\chi_{m 2}}=64 s_{1}^{-2} s_{2} c_{\delta}^{2},
\end{array}
$$

for all $t \in \mathbb{R}^{+}$. As described in the Appendix, we can select the function between the gains as

$$
\sigma(t)=c_{\sigma} t^{2}, \quad c_{\sigma}=\frac{c_{\chi_{m 1}}^{-2}+c_{\chi_{m 2}}}{2}>0,
$$

for all $t \in \mathbb{R}^{+}$. Simple computations in Steps 4) and 5) show that the decay rate is given by

$$
\beta(s, t)=s \sqrt{c_{1}} \exp \left(-\frac{c_{2}}{c_{1}} t\right),
$$


for all $s, t \in \mathbb{R}^{+}$and with the positive constants:

$$
\begin{aligned}
c_{1} & =\frac{8 \max \left\{s_{2}, c_{\sigma}\right\}}{\min \left\{s_{1}, c_{\sigma}\right\}}, \\
c_{2} & =\frac{1}{4} \min \left\{2(k-1) c_{\rho},\left(\theta-b_{2}\right) s_{1} s_{2}^{-1}\right\} .
\end{aligned}
$$

Furthermore, the constant of the practical stability is

$$
\begin{aligned}
c= & 16\left(\frac{c_{1}}{s_{1} \min \left\{s_{1}, c_{\sigma}\right\}}\right)^{\frac{1}{2}}\left(\left|d_{y}\right|_{\infty}+2 c_{\delta}\left|d_{\psi}\right|_{\infty}\right)^{\frac{1}{2}} \\
& +64 c_{\rho}^{-1}\left(\frac{c_{1} c_{\sigma}}{\min \left\{s_{1}, c_{\sigma}\right\}}\right)^{\frac{1}{2}}\left(\left|d_{y}\right|_{\infty}+\left|\dot{d}_{y}\right|_{\infty}+\left|\dot{d}_{\psi}\right|_{\infty}\right) .
\end{aligned}
$$

\section{B. Systems with additive triangular nonlinearity}

Let us now consider the constant matrices $A \in \mathbb{R}^{n}$ and $C \in \mathbb{R}^{1 \times n}$ given by

$$
A=\left(\begin{array}{cccc}
0 & 1 & & 0 \\
& \ddots & \ddots & \\
& & \ddots & 1 \\
0 & & & 0
\end{array}\right), \quad C=\left(\begin{array}{llll}
1 & 0 & \ldots & 0
\end{array}\right)
$$

and suppose that $\zeta: \mathbb{R}^{n} \times \mathbb{R}^{m} \rightarrow \mathbb{R}^{n}$ is a nonlinear and continuous function of the form

$$
\zeta(x, u)=\left(\begin{array}{c}
\zeta_{1}\left(x_{1}, u\right) \\
\zeta_{2}\left(x_{1}, x_{2}, u\right) \\
\vdots \\
\zeta_{n}(x, u)
\end{array}\right)
$$

for all $x=\left(x_{1}, \ldots, x_{n}\right) \in \mathbb{R}^{n}$ and $u \in \mathbb{R}^{m}$. Our goal is to estimate the states of the system:

$$
\left\{\begin{array}{l}
\dot{x}=A x+\zeta(x, u) \\
y_{\psi}=\psi(y)+d_{\psi}
\end{array}\right.
$$

where $y=C x+d_{y}$ and where $\psi: \mathbb{R} \rightarrow \mathbb{R}$ satisfies Assumption 2. As in the previous case, we need to add an assumption on $\zeta$. To simplify the notation, we define

$$
\underline{x}_{i}=\left(x_{1}, \ldots, x_{i}\right) \in \mathbb{R}^{i},
$$

for each $x \in \mathbb{R}^{n}$ and each $i=1, \ldots, n$.

Assumption 6: The function $\zeta$ is coordinate-wise Lipschitz continuous in the first entrance. That is, for each $i=1, \ldots, n$, there exists a positive constant $c_{\zeta i}$ such that

$$
\left|\zeta_{i}\left(\underline{x}_{i}, u\right)-\zeta_{i}\left(\underline{\hat{x}}_{i}, u\right)\right| \leq c_{\zeta i}\left|\underline{x}_{i}-\underline{\hat{x}}_{i}\right|,
$$

for all $x, \hat{x} \in \mathbb{R}^{n}$ and all $u \in \mathbb{R}^{m}$.

As opposed to the state-affine case, the nonlinearity $\zeta$ in system (36) depends on the full-state $x$ which complicates its estimation. The following observer was introduced in [8] and plays the role of observer (2):

$$
\left\{\begin{array}{l}
\dot{\hat{x}}=A \hat{x}+\zeta(\hat{x}, u)+S_{\infty}^{-1} C^{\prime}(y-C \hat{x}) \\
0=-\theta S_{\infty}-A^{\prime} S_{\infty}-S_{\infty} A+C^{\prime} C
\end{array}\right.
$$

where $\theta>0$ is a tuning parameter. As before, $S_{\infty}$ is symmetric and positive definite. Let us denote the eigenvalue extrema of $S_{\infty}$ by

$$
s_{1}=\lambda_{\min }\left(S_{\infty}\right), \quad s_{2}=\lambda_{\max }\left(S_{\infty}\right)
$$

and define $S_{\infty, 1}$ as the solution of the second equation in (37) corresponding to the unitary tuning, that is,

$$
S_{\infty, 1}=\theta^{-1} \Delta S_{\infty} \Delta
$$

where

$$
\Delta=\left(\begin{array}{lll}
\theta & & 0 \\
& \ddots & \\
0 & & \theta^{n}
\end{array}\right) .
$$

Finally, we name the following maxima:

$$
c_{\zeta}=\max _{i=1, \ldots, n} c_{\zeta i}, \quad s_{m}=\max _{i, j=1, \ldots, n}\left|\left(S_{\infty, 1}\right)_{i, j}\right| .
$$

We next show that observer (37) satisfies Assumption 1 by using similar techniques to those of [8]. In this case, the input set $\mathcal{U}$ can be taken simply as the set of continuous functions $u: \mathbb{R}^{+} \rightarrow \mathbb{R}^{m}$. We define the function $\bar{V}: \mathbb{R}^{n} \rightarrow \mathbb{R}^{+}$as the norm induced by $S_{\infty}$, that is,

$$
\begin{aligned}
\bar{V}(e) & =\left(e^{\prime} S_{\infty} e\right)^{\frac{1}{2}} \\
& =|e|_{S_{\infty}},
\end{aligned}
$$

for all $e \in \mathbb{R}^{n}$. Clearly, we can select the class $\mathcal{K}_{\infty}$ functions given by

$$
\bar{\alpha}_{1}(t)=s_{1}^{\frac{1}{2}} t, \quad \bar{\alpha}_{2}(t)=s_{2}^{\frac{1}{2}} t
$$

for all $t \in \mathbb{R}^{+}$. Moreover, the following holds for all $t \in \mathbb{R}^{+}$, $e \in \mathbb{R}^{n}-\{0\}$ and $d \in \mathbb{R}$. The function $\mathcal{F}$ from (5) takes the form

$$
\begin{aligned}
\mathcal{F}(t, e, d)= & A e-S_{\infty}^{-1} C^{\prime}(C e+d) \\
& +\zeta(x(t), u(t))-\zeta(x(t)-e, u(t)) .
\end{aligned}
$$

It then follows that

$$
\begin{aligned}
\frac{\partial \bar{V}}{\partial e}(e) \mathcal{F}(t, e, d) & =\frac{1}{2}|e|_{S_{\infty}}^{-1}\left(2 e^{\prime} S_{\infty} \mathcal{F}(t, e, d)\right) \\
& \leq-\frac{\theta}{2}|e|_{S_{\infty}}-|e|_{S_{\infty}}^{-1} e^{\prime} C^{\prime} d \\
+|e|_{S_{\infty}}^{-1} e^{\prime} S_{\infty} \cdot & (\zeta(x(t), u(t))-\zeta(x(t)-e, u(t))) \\
& \leq-\frac{\theta}{2}|e|_{S_{\infty}}+s_{1}^{-\frac{1}{2}}|C||d| \\
+\mid \zeta(x(t), u(t)) & -\left.\zeta(x(t)-e, u(t))\right|_{S_{\infty}} .
\end{aligned}
$$

On the other hand, computing the induced norm and using (38) we have

$$
\begin{aligned}
\mid \zeta(x(t), u(t))- & \left.\zeta(x(t)-e, u(t))\right|_{S_{\infty}} ^{2}= \\
& \sum_{i, j=1}^{n} \frac{\theta}{\theta^{i+j}}\left(S_{\infty, 1}\right)_{i, j} \bar{\zeta}(i, t, e) \bar{\zeta}(j, t, e),
\end{aligned}
$$

where $\bar{\zeta}(i, t, e)=\zeta_{i}\left(\underline{x}_{i}(t), u(t)\right)-\zeta_{i}\left(\underline{x}_{i}(t)-\underline{e}_{i}, u(t)\right)$. Therefore, Assumption 6 implies:

$$
\begin{array}{r}
|\zeta(x(t), u(t))-\zeta(x(t)-e, u(t))|_{S_{\infty}}^{2} \leq \sum_{i, j=1}^{n} \frac{\theta s_{m} c_{\zeta}^{2}}{\theta^{i+j}}\left|\underline{e}_{i}\right|\left|\underline{e}_{j}\right| \\
\leq n^{2} s_{m} c_{\zeta}^{2} \lambda_{\min }\left(S_{\infty, 1}\right)^{-1}|e|_{S_{\infty}}^{2},
\end{array}
$$


where we used the inequality $\left|\theta^{-i} \underline{e}_{i}\right| \leq\left|\Delta^{-1} e\right|$. Putting (40) and (41) together,

$$
\begin{aligned}
\frac{\partial \bar{V}}{\partial e}(e) \mathcal{F}(t, e, d) \leq & -\frac{\theta}{2}|e|_{S_{\infty}}+s_{1}^{-\frac{1}{2}}|C||d| \\
& +n s_{m}^{\frac{1}{2}} c_{\zeta} \lambda_{\min }\left(S_{\infty, 1}\right)^{-\frac{1}{2}}|e|_{S_{\infty}} \\
\leq & -\bar{\alpha}_{3}(|e|),
\end{aligned}
$$

whenever $|e| \geq \bar{\chi}(|d|)$ and where:

$$
\bar{\chi}(t)=s_{1}^{-1} t, \quad \bar{\alpha}_{3}(t)=\frac{s_{1}^{\frac{1}{2}}}{2}(\theta-b) t,
$$

for all $t \in \mathbb{R}^{+}$and for

$$
\theta>b=2\left(n s_{m}^{\frac{1}{2}} c_{\zeta} \lambda_{\min }\left(S_{\infty, 1}\right)^{-\frac{1}{2}}+1\right) .
$$

Notice that such $\theta$ exists since $S_{\infty, 1}$ corresponds to the unitary tuning.

In order to construct the new observer (11) and given that we are in the case of Remark 4, we can choose $\alpha$ as the identity function and:

$$
\begin{aligned}
\varphi(\hat{x}, u) & \geq c_{\varphi}(|A \hat{x}+\zeta(\hat{x}, u)|+1), \\
\rho(t) & =17\left(s_{1}^{-3} s_{2}\right)^{\frac{1}{2}} \delta(4 t)
\end{aligned}
$$

for some $c_{\varphi} \geq 1$ and all $\hat{x} \in \mathbb{R}^{n}, u \in \mathbb{R}^{m}$ and $t \in \mathbb{R}^{+}$. The new observer (11) then takes the form:

$$
\left\{\begin{aligned}
\dot{\hat{x}}= & A \hat{x}+\zeta(\hat{x}, u)+S_{\infty}^{-1} C^{\prime}(\hat{y}-C \hat{x}) \\
\dot{\hat{y}}= & \frac{\partial \psi}{\partial y}(\hat{y})^{-1} \frac{\partial \psi}{\partial y}(C \hat{x}) C(A \hat{x}+\zeta(\hat{x}, u)) \\
& +\frac{\partial \psi}{\partial y}(\hat{y})^{-1} \varphi(\hat{x}, u) K\left(y_{\psi}-\psi(\hat{y})\right),
\end{aligned}\right.
$$

where $S$ is as in (37) and $K$ as in (14) with $k>1$.

Corollary 2 (triangular case): Let Assumptions 2, 3 and 6 hold and consider systems (36) and (42). For any tuning

$$
\theta>2\left(n s_{m}^{\frac{1}{2}} c_{\zeta} \lambda_{\min }\left(S_{\infty, 1}\right)^{-\frac{1}{2}}+1\right),
$$

where $c_{\zeta}$ and $s_{m}$ are as in (39), there exist a function $\beta \in \mathcal{K} \mathcal{L}$ and a constant $c \geq 0$ such that for all $x(0), \hat{x}(0) \in \mathbb{R}^{n}$, all $\hat{y}(0) \in \mathbb{R}$ and all $u \in \mathcal{U}$ the estimation errors $e=x-\hat{x}$ and $\xi=y_{\psi}-\psi(\hat{y})$ are defined on $\mathbb{R}^{+}$and

$$
|(\xi(t), e(t))| \leq \beta(|(\xi(0), e(0))|, t)+c, \quad \forall t \geq 0 .
$$

Moreover, if the disturbances $d_{y}$ and $d_{\psi}$ are both zero then $c$ is zero as well.

Proof: It follows from our previous development and from Theorem 3. Indeed, we showed that Assumption 1 is satisfied by using the specific forms of $A$ and $C$ and by using Assumption 6.

Remark 7: If the states of system (36) are uniformly bounded and if $\zeta$ is of class $C^{1}$ then Assumption 6 can be met by saturating $\zeta$. Indeed, denote by $X$ the state space and let $\mu>0$ be such that $|x| \leq \mu$, for all $x \in X$. Set each $\zeta_{i}^{s}: \mathbb{R}^{i} \times \mathbb{R}^{m} \rightarrow \mathbb{R}$ as

$$
\zeta_{i}^{s}\left(x_{1}, \ldots, x_{i}, u\right)=\zeta_{i}\left(\mu \operatorname{sat}\left(\mu^{-1} x_{1}\right), \ldots, \mu \operatorname{sat}\left(\mu^{-1} x_{i}\right), u\right),
$$

where sat $: \mathbb{R} \rightarrow \mathbb{R}$ is given by $\operatorname{sat}(t)=\min \{1,|t|\} \operatorname{sign}(t)$. Replacing $\zeta$ by $\zeta^{s}$ defines an equivalent system and the latter function satisfies Assumption 6. We can proceed similarly for $\psi$ and Assumption 3 but using a non-constant and smooth saturation.

\section{Numerical Results}

In this section, we study specific cases of systems (24) and (36) in order to provide numerical examples of Corollaries 1 and 2.

\section{A. Example for state-affine systems}

Let us suppose that system (24) is given by:

$$
A(u)=\left(\begin{array}{ll}
0 & u \\
0 & 0
\end{array}\right), \quad \eta(y, u)=\left(\begin{array}{c}
\sin (y) \\
u^{2}
\end{array}\right), \quad C=\left(\begin{array}{ll}
1 & 0
\end{array}\right),
$$

for all $y, u \in \mathbb{R}$. The input and the nonlinear function are defined as:

$$
u(t)=\cos (t), \quad \psi(y)=\sin (y)+2 y .
$$

The function $\psi$ is a diffeomorphism whose inverse has no closed form, and it is straight-forward to check that Assumptions 2, 3 and 4 are satisfied. Moreover, we can select $\delta$ as the identity function and it can be shown that $u$ is regularly persistent, see for example [49]. The lower bound of $\theta$ in Corollary 1 has a value of 6 when $S(0)=I_{2}$. A simple computation shows that, according to (31) and (33), we can select

$$
\varphi(\hat{x}, u) K(\xi)=r\left(\left|\hat{x}_{2}\right|+1\right) \cdot \xi,
$$

for all $\hat{x} \in \mathbb{R}^{2}$ and $u, \xi \in \mathbb{R}$ and where $r>0$ is to be tuned.

We initialize the system and the observers at: $x(0)=$ $(10,0)^{\prime}, \bar{x}(0)=\hat{x}(0)=(4,9)^{\prime}$ and $\hat{y}(0)=C \hat{x}(0)$ and we set the tuning parameter $\theta=7$. The results are shown in Figures 2 and 3. The left and right columns represent the tunings $r=0.5$ and $r=2$. We simulate two disturbance cases: (i) $d_{y}$ and $d_{\psi}$ are both zero (Figure 2), (ii) $d_{y}$ and $d_{\psi}$ are uniformly distributed numbers respectively between \pm 0.8 and \pm 1.3 (Figure 3 ).

Figure 2: In the top two rows, we can see that observer (25), which uses $y$, correctly estimates the states of the system. As also depicted in the top two rows, the new observer (34) achieves at most the performance of observer (25). Moreover, the performance is closer for the larger choice of $r$ (right column). Finally, the quality of the state estimation given by the new observer (34) depends of the estimation of $y$ by $\hat{y}$, as can be seen in the third row.

Figure 3: In the top two rows, we can see that the state estimation from observer (25) converges to a neighborhood of the system state, as expected from a DES observer. The performance of observer (25) and the new observer (34) are again similar for the larger $r$. Moreover, the new observer (34) outperforms observer (25) in terms of noise robustness. This is not surprising since noise amplification is a common problem for high-gain observers when their tuning parameter $\theta$ is large [12]. The noise, in contrast, is averaged out by the definition of $\hat{y}$.

These figures show that, for $r$ large enough, the new observer in (34) recovers the performance of observer (25). Furthermore, the design of the new observer (34) seems to be more robust against measurement noise. 

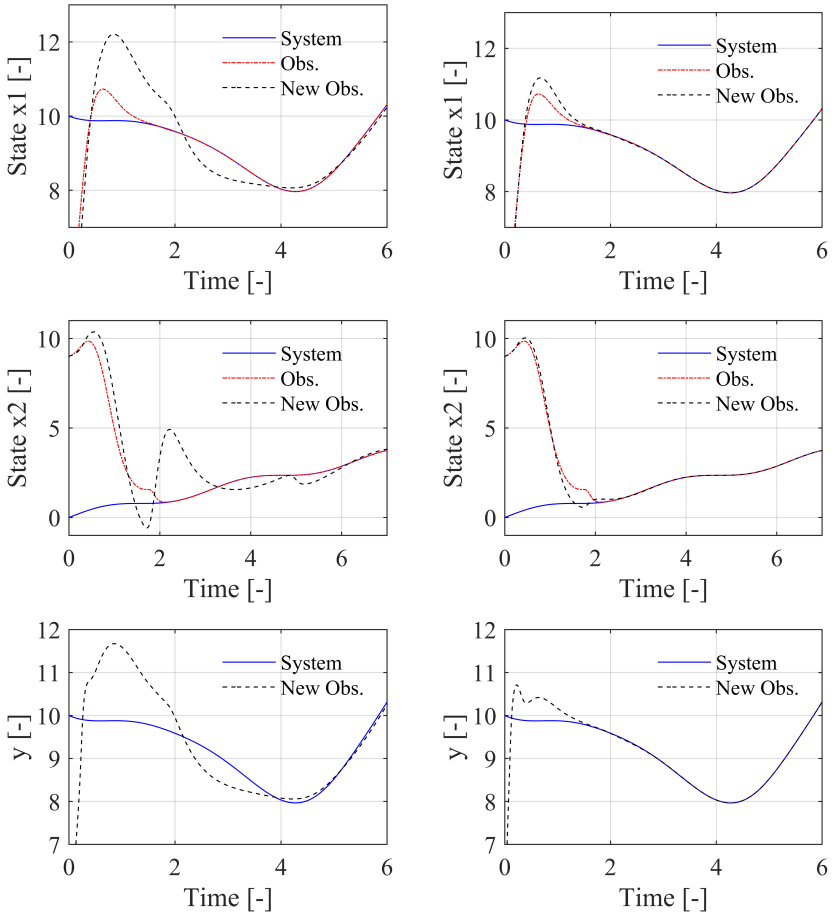

Figure 2. Comparison of the new observer with the observer that uses $y$ in the case: $d_{y}=0, d_{\psi}=0$. Estimation of the system states of (24) (blue solid) by observer (25) (red pointed-dashed) and by the new observer (34) (black dashed). The columns correspond to different tunings of $r$ in (43).

\section{B. Example for systems with triangular nonlinearity}

The authors in [26] consider systems in the form of (36) and illustrate the case given by:

$$
\begin{gathered}
A=\left(\begin{array}{ll}
0 & 1 \\
0 & 0
\end{array}\right), \quad C=\left(\begin{array}{ll}
1 & 0
\end{array}\right), \\
\zeta(x, u)=\left(\begin{array}{c}
0 \\
-x_{1}-2 x_{2}+a x_{1}^{2} x_{2}+u
\end{array}\right),
\end{gathered}
$$

for all $x \in \mathbb{R}^{2}$ and $u \in \mathbb{R}$ and for some $a>0$. They also define the input and the nonlinear output as:

$$
u(t)=b \sin (2 t), \quad \psi(y)=\frac{1}{3} y^{3}-\frac{1}{2} y^{2}+y,
$$

for some $b>0$. As opposed to [26], we consider disturbances. Notice that $\psi$ is a diffeomorphism whose derivative is bounded from below by 0.75 and, therefore, Assumption 2 is met. However, $\psi$ does not satisfy Assumption 3 and $\zeta$ satisfies only locally Assumption 6. Hence, we make use of the following.

As in [4] or [26], for $a=0.25$ and $b=0.2$ every state starting at

$$
X=\left\{x \in \mathbb{R}^{2} \mid 1.5 x_{1}^{2}+x_{1} x_{2}+0.5 x_{2}^{2} \leq \sqrt{2}\right\}
$$

remains in that set for all positive times. Therefore, in Remark 7 we can replace $\psi$ and $\zeta$ by their saturated versions $\psi^{s}$ and $\zeta^{s}$. These new functions satisfy Assumptions 2, 3 and 6. Similar to the first case, we set

$$
\varphi(\hat{x}, u) K(\xi)=r\left(\left|\hat{x}_{1}\right|+\left|\hat{x}_{2}\right|+\left|\hat{x}_{1}^{2} \hat{x}_{2}\right|+1\right) \cdot \xi
$$
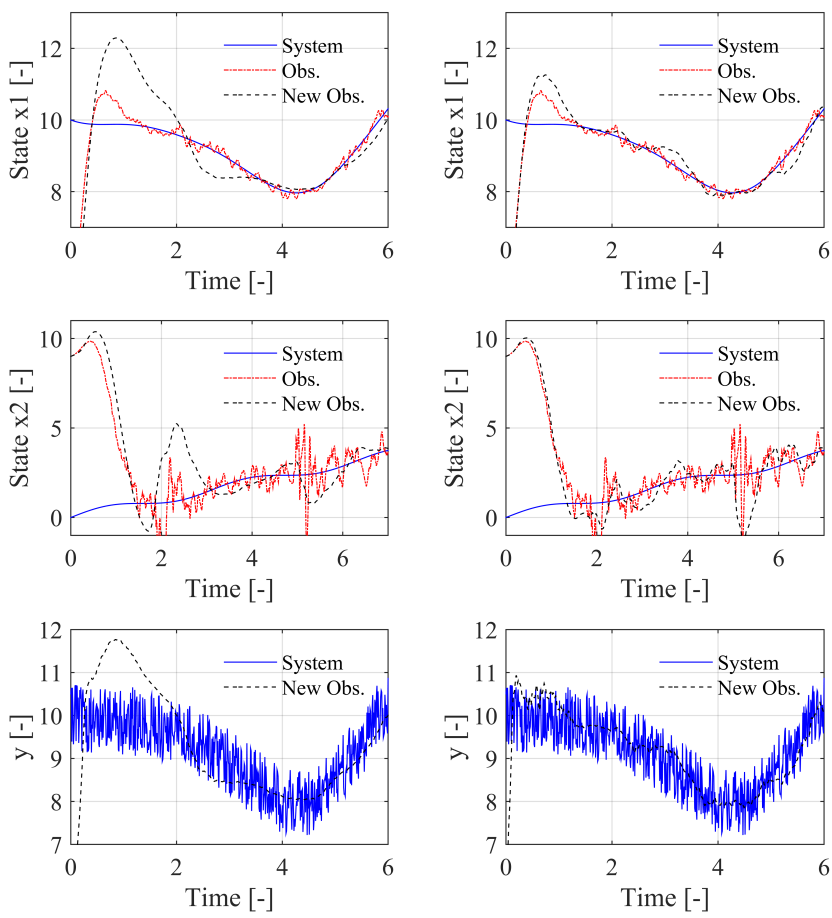

Figure 3. Comparison of the new observer with the observer that uses $y$ in the case: $d_{y} \neq 0, d_{\psi} \neq 0$. Estimation of the system states of (24) (blue solid) by observer (25) (red pointed-dashed) and by the new observer (34) (black dashed). The columns correspond to different tunings of $r$ in (43).

for all $\hat{x} \in \mathbb{R}^{2}$ and $u, \xi \in \mathbb{R}$ and where $r>0$. On the other hand, the observer from [26] is given by

$$
\dot{\hat{z}}=A \hat{z}+\left(\begin{array}{c}
0 \\
\zeta_{0}(\hat{z}, u)
\end{array}\right)+\left(\begin{array}{l}
(8 / 3) \epsilon^{-1} \\
(4 / 3) \epsilon^{-2}
\end{array}\right)\left(y_{\psi}-\psi(C \hat{z})\right),
$$

where $\zeta_{0}(\hat{z}, u)=-\hat{z}_{1}-2 \hat{z}_{2}+a \operatorname{sat}\left(\hat{z}_{1}^{2} \hat{z}_{2}\right)+u, y_{\psi}=\psi(C x+$ $\left.d_{y}\right)+d_{\psi}$ and where $\epsilon>0$ is to be tuned.

In order to make a fair comparison of observers (42) and (44): first we choose two far enough values of $\epsilon$, then we try to improve or to match this performance by tuning $\theta$ and $r$.

We initialize the system and the observers at: $x(0)=$ $(1,-1)^{\prime}, \hat{x}(0)=\hat{z}(0)=(0,0)^{\prime}$ and $\hat{y}=C \hat{x}(0)$ and we fix the value $r=20$. The results can be seen in Figures 4 and 5. The left column represents the tunings $\theta=2$ and $\epsilon=0.5$ while the right column corresponds to $\theta=9$ and $\epsilon=0.11$. We simulate two cases: (i) $d_{y}$ and $d_{\psi}$ are both zero (Figure 4 ), (ii) $d_{y}$ and $d_{\psi}$ are uniformly distributed numbers respectively between \pm 0.3 and \pm 0.4 (Figure 5).

Figure 4: In the top two rows, we see that both the new observer (42) and observer (44) render quite similar estimations that converge to the system states. Furthermore, higher tuning of $\theta$ and $\epsilon^{-1}$ leads to faster state reconstruction at the price of higher peaking (right column). The third row shows that tuning $\theta$ does not have a very strong effect in the estimation of $y$ given by $\hat{y}$.

Figure 5: The top two rows show that both observers (42) and (44) provide similar estimations, this time converging to neighborhoods of the states. Increasing the value of $\epsilon^{-1}$ in observer (44) amplifies the noise, this is slightly less visible 

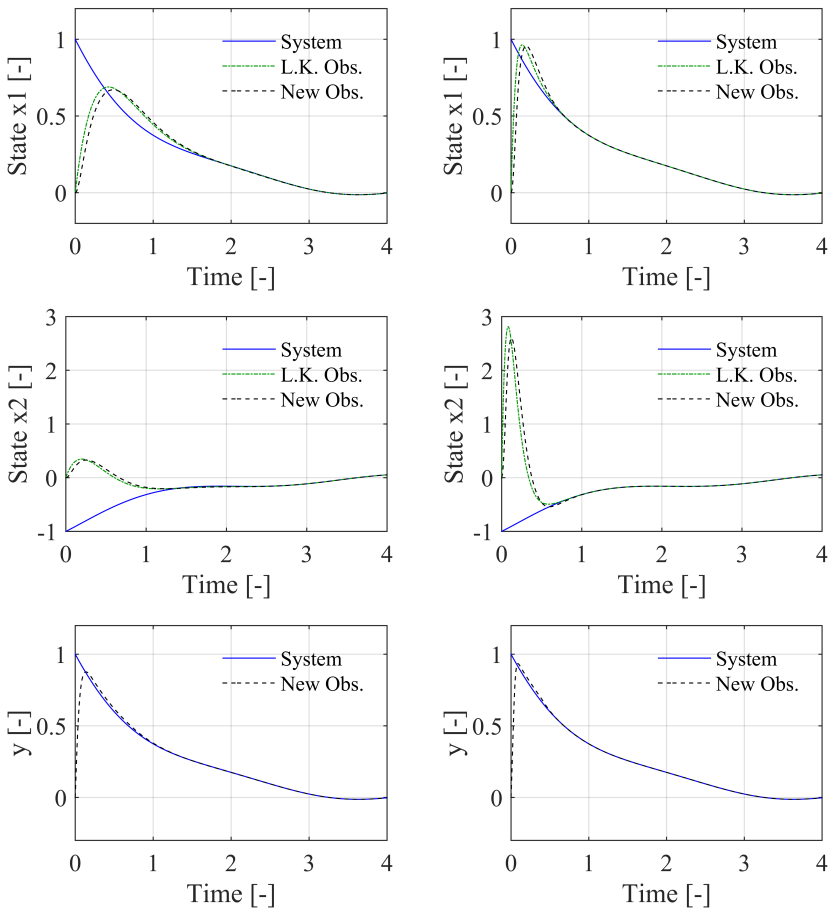

Figure 4. Comparison of the new observer with the observer of J. Lei and H.K. Khalil [26] in the case: $d_{y}=0, d_{\psi}=0$. Estimation of the system states in (36) (blue solid) by observer (44) (green pointed-dashed) and by the new observer (42) (black dashed). The columns correspond to different tunings of $\theta$ and $\epsilon$.

for the larger choice of $\theta$ and the new observer (42) (right column).

These figures suggest that both observers have similar performances if they are properly tuned. It is clear that observer (44) has a simpler design. Nevertheless, our methodology provides a new observer design for a much more general family of systems.

\section{CONCLUSION}

The method presented in this work concerns observer redesign for nonlinear systems in the presence of output transformations. The new observer consists in an interconnection of the initial observer dynamics with an estimator of the unavailable output. By using the small-gain theorem, we showed that the new observer converges asymptotically to a neighborhood of zero that depends on the amplitude of the disturbances and their derivatives. We then studied the new observer design for two major families of systems that differ in their observability properties. The simulations showed that the new observer recovers the performance of the initial observer and that its performance is comparable to that of other related observers. Unlike previous studies, the generality of our approach considers systems without a specific form at the cost of a more intricate design. Future work will focus on relaxing the conditions on the initial observer by considering weaker versions of the DES property, such as quasi-DES developed in [32].
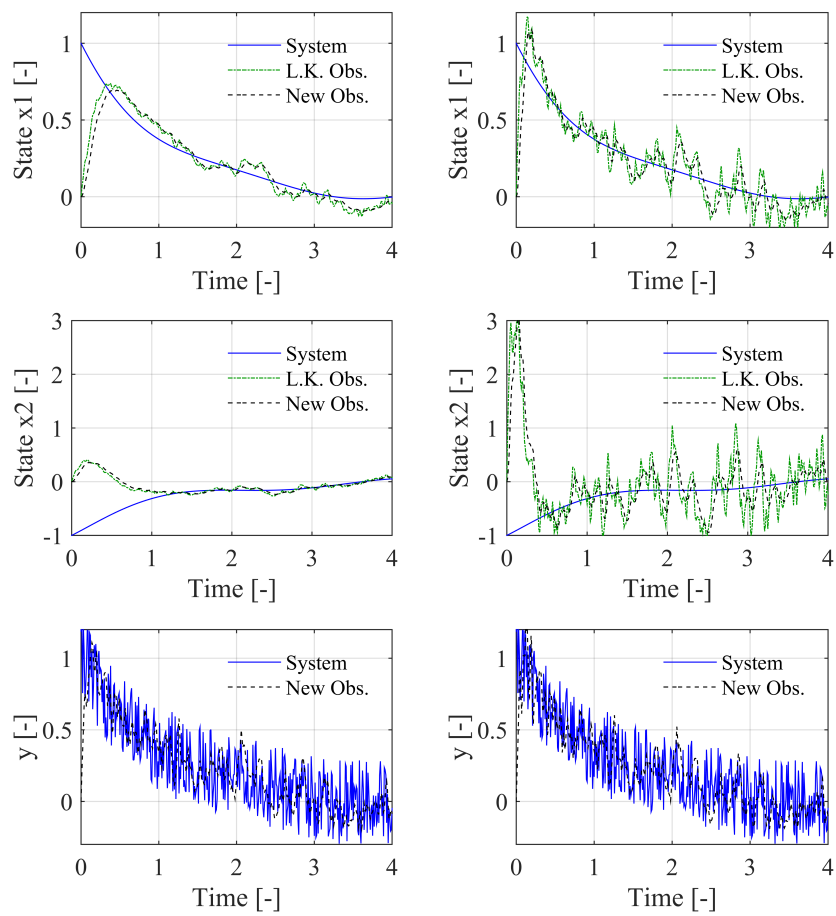

Figure 5. Comparison of the new observer with the observer of J. Lei and H.K. Khalil [26] in the case: $d_{y} \neq 0, d_{\psi} \neq 0$. Estimation of the system states in (36) (blue solid) by observer (44) (green pointed-dashed) and by the new observer (42) (black dashed). The columns correspond to different tunings of $\theta$ and $\epsilon$.

\section{APPENDIX}

\section{A. Proof of Theorem 1 (Lyapunov function)}

This proof is partly based on a known comparison principle we next state.

Lemma 3 ([50]): For any $\hat{\alpha} \in \mathcal{K}$ there exists $\hat{\beta} \in \mathcal{K} \mathcal{L}$ with the following property. For every $r \in(0, \infty]$ and for every locally Lipschitz function $\zeta:[0, r) \rightarrow \mathbb{R}^{+}$the inequality

$$
\begin{aligned}
& \dot{\zeta}(t) \leq-\hat{\alpha}(\zeta(t)), \text { for a.e. } t \in[0, r) \text { implies } \\
& \zeta(t) \leq \hat{\beta}(\zeta(0), t), \text { for every } t \in[0, r) .
\end{aligned}
$$

Moreover, if we define $\eta(s)=-\int_{1}^{s} \frac{d t}{\min \{t, \hat{\alpha}(t)\}}$ then we can select

$$
\hat{\beta}(s, t)=\left\{\begin{array}{l}
\eta^{-1}(\eta(s)+t), \text { if } s>0 \\
0, \text { if } s=0 .
\end{array}\right.
$$

Proof of Theorem 1: Fix for the moment $(z, w) \in \mathcal{G}$ and define the function $V^{z}=V(\cdot, z)$ and the subset

$$
S=\left\{t \in\left[0, T_{z, w}\right) \mid V^{z}(t) \leq \Gamma(t)\right\},
$$

where

$$
\Gamma(t)=\alpha_{2}\left(\chi\left(\sup _{s \in[0, t]}|w(s)|\right)+c_{L}\right) .
$$

First, we prove by contradiction that for all $t_{0} \in \mathbb{R}^{+}$:

$$
t_{0} \in S \text { implies } t \in S, \quad \forall t \in\left[t_{0}, T_{z, w}\right) .
$$

If this is not the case, then there is $t_{0} \in S$ such that

$$
t^{*}=\inf \left\{t \in\left[t_{0}, T_{z, w}\right) \mid V^{z}(t) \geq \Gamma(t)+\epsilon\right\}<\infty,
$$


where $\epsilon>0$ is small enough. Notice that the continuity of $V^{z}$ and property (7) imply that $t^{*}>t_{0}$ and that $\alpha_{2}\left(\left|z\left(t^{*}\right)\right|\right)>$ $\Gamma\left(t^{*}\right)$. In particular, $z\left(t^{*}\right) \neq 0$. It follows from the definition of $\Gamma$ in (45) and from property (8) that

$$
\dot{V}^{z}(t) \leq-\alpha_{3}(|z(t)|),
$$

for a.e. $t \in B_{\epsilon_{1}}\left(t^{*}\right) \subseteq\left[t_{0}, T_{z, w}\right)-z^{-1}(0)$ and where $\epsilon_{1}$ is a small enough radius. This means that $V^{z}$ does not increase on $B_{\epsilon_{1}}\left(t^{*}\right)$, which contradicts the choice of $t^{*}$. We conclude that our assumption in (46) must be true.

Let us now define

$$
s^{*}=\inf S \leq \infty
$$

and assume for now that $s^{*}$ is finite. Notice that $\left[s^{*}, T_{z, w}\right) \subseteq$ $S$, indeed, this is a consequence of $s^{*} \in S$ and of the claim shown in (46). Therefore, property (7) implies that

$$
\begin{aligned}
|z(t)| & \leq \gamma\left(\sup _{s \in[0, t]}|w(s)|\right)+c \\
& =\sup _{s \in[0, t]} \gamma(|w(s)|)+c,
\end{aligned}
$$

for all $t \in\left[s^{*}, T_{z, w}\right)$ and where

$$
\gamma=\alpha_{1}^{-1}\left(2 \alpha_{2}(2 \chi)\right) \in \mathcal{K}, \quad c=\alpha_{1}^{-1}\left(2 \alpha_{2}\left(2 c_{L}\right)\right) \geq 0 .
$$

On the other hand, it is clear that $S \cap\left[0, s^{*}\right)$ is empty and, as a consequence, also $z^{-1}(0) \cap\left[0, s^{*}\right)$. Then, properties (7) and (8) provide

$$
\dot{V}^{z}(t) \leq-\hat{\alpha}\left(V^{z}(t)\right)
$$

for a.e. $t \in\left[0, s^{*}\right)$ and where $\hat{\alpha}=\alpha_{3}\left(\alpha_{2}^{-1}\right) \in \mathcal{K}$. We can then apply Lemma 3 to get a function $\hat{\beta} \in \mathcal{K} \mathcal{L}$ such that $V^{z}(t) \leq \hat{\beta}\left(V^{z}(0), t\right)$, for all $t \in\left[0, s^{*}\right)$. Hence, property (7) implies that

$$
|z(t)| \leq \beta(|z(0)|, t)
$$

for all $t \in\left[0, s^{*}\right)$ and where

$$
\beta(s, t)=\alpha_{1}^{-1}\left(\hat{\beta}\left(\alpha_{2}(s), t\right)\right),
$$

for all $s, t \in \mathbb{R}^{+}$. It is straight-forward to check that $\beta \in \mathcal{K} \mathcal{L}$. We conclude the desired inequality in (6) by joining inequalities (47) and (48).

We proceed similarly if $s^{*}$ is infinite. The only difference being that inequality (48) is satisfied on the whole interval $\left[0, T_{z, w}\right)$. Finally, notice that $\beta, \gamma$ and $c$ are independent from the choice of $(z, w)$.

\section{B. Proof of Theorem 2 (small-gain)}

Lemma 4 ([35]): Consider functions $\sigma_{1} \in \mathcal{K}$ and $\sigma_{2} \in \mathcal{K}_{\infty}$ and suppose that $\sigma_{1}(t)<\sigma_{2}(t)$, for all $t>0$. Then, there exists a function $\sigma \in \mathcal{K}_{\infty}$ that is continuously differentiable on $t>0$ and that satisfies:

$$
0<\dot{\sigma}(t), \quad \sigma_{1}(t)<\sigma(t)<\sigma_{2}(t), \quad \forall t>0 .
$$

The fact that the derivative of $\sigma$ is strictly positive on $t>0$ is crucial. This is not necessarily true for an arbitrary function $\mathcal{K}_{\infty} \cap C^{1}$ as shown in [41]. Notice that the function $\sigma$ at zero is no more than continuous and that it appears in the definition of $V$ below. This does not cause any problems given our specific definition of Lyapunov function.

Proof of Theorem 2: The small-gain condition in (10) implies the existence of $\sigma$ as in Lemma 4, that is:

$$
0<\dot{\sigma}(t), \quad \chi_{m 2}(t)<\sigma(t)<\chi_{m 1}^{-1}(t), \quad \forall t>0 .
$$

By Theorem 1, it suffices to find a Lyapunov function for the family $\mathcal{G} \times\{0\}$. Consider the Lyapunov functions $V_{1}$ and $V_{2}$, respectively for $\mathcal{G}$ and for $\mathcal{G}^{-1}$, and set

$$
V(t,(z, w))=\max \left\{\sigma\left(V_{1}(t, z)\right), V_{2}(t, w)\right\},
$$

for all $t \in \mathbb{R}^{+}$and $(z, w) \in \mathbb{R}^{n_{1}} \times \mathbb{R}^{n_{2}}$. Let us fix for the moment any $(z, w) \in \mathcal{G}$ and, as before, the notation $V_{1}^{z}, V_{2}^{w}$ and $V^{z, w}$ indicates evaluation on the corresponding trajectories.

It is clear that $V^{z, w}$ is everywhere continuous. Moreover, $V_{1}^{z}$ and $V_{2}^{w}$ are zero precisely where their corresponding trajectories are zero. Hence, $V^{z, w}$ is also locally Lipschitz on $I_{z, w}-\left(z^{-1}(0) \cup w^{-1}(0)\right)$. We consider the following partition of the interval $I_{z, w}$ :

$$
\begin{aligned}
D & =\left\{t \in I_{z, w}: \sigma\left(V_{1}^{z}(t)\right)=V_{2}^{w}(t)\right\}, \\
D_{z} & =\left\{t \in I_{z, w}: \sigma\left(V_{1}^{z}(t)\right)>V_{2}^{w}(t)\right\}, \\
D_{w} & =\left\{t \in I_{z, w}: \sigma\left(V_{1}^{z}(t)\right)<V_{2}^{w}(t)\right\},
\end{aligned}
$$

in order to write

$$
V^{z, w}(t)=\left\{\begin{array}{l}
\sigma\left(V_{1}^{z}(t)\right), \text { if } t \in D_{z} \\
V_{2}^{w}(t), \text { if } t \in D_{w} \cup D .
\end{array}\right.
$$

The open subsets $D_{z}$ and $D_{w}$ of $I_{z, w}$ are contained respectively in $I_{z, w}-z^{-1}(0)$ and $I_{z, w}-w^{-1}(0)$. In particular, this implies that $V^{z, w}$ is also locally Lipschitz on $D_{z} \cup D_{w}$ and, consequently, on the whole $I_{z, w}-(z, w)^{-1}(0)$ as needed.

The following verifies property (7) of $V^{z, w}$ by using:

$$
\begin{aligned}
& \alpha_{1}=\frac{1}{2} \min \left\{\sigma\left(\alpha_{11}\left(\frac{\cdot}{2}\right)\right), \alpha_{21}\left(\frac{\cdot}{2}\right)\right\} \in \mathcal{K}_{\infty}, \\
& \alpha_{2}=\max \left\{\sigma\left(\alpha_{12}\right), \alpha_{22}\right\} \in \mathcal{K}_{\infty} .
\end{aligned}
$$

The corresponding property of $V_{1}^{z}$ and $V_{2}^{w}$ implies:

$$
\begin{aligned}
\alpha_{1}(|(z(t), w(t))|) & \leq \alpha_{1}(2|z(t)|)+\alpha_{1}(2|w(t)|) \\
& \leq \max \left\{\sigma\left(V_{1}^{z}(t)\right), V_{2}^{w}(t)\right\} \\
& \leq \max \left\{\sigma\left(\alpha_{12}(|z(t)|)\right), \alpha_{22}(|w(t)|)\right\} \\
& \leq \alpha_{2}(|(z(t), w(t))|),
\end{aligned}
$$

for all $t \in \mathbb{R}^{+}$. We now continue by checking property (8) of $V^{z, w}$. For this purpose, we select the function

$$
\alpha_{3}=\frac{1}{2} \min \left\{\hat{\alpha}_{1}, \hat{\alpha}_{2}\right\} \in \mathcal{K},
$$

where $\hat{\alpha}_{1}(0)=0$ and

$$
\begin{aligned}
& \hat{\alpha}_{1}=\dot{\sigma}\left(\sigma^{-1}\left(\alpha_{1}\right)\right) \cdot \alpha_{13}\left(\alpha_{12}^{-1}\left(\sigma^{-1}\left(\alpha_{1}\right)\right)\right), \\
& \hat{\alpha}_{2}=\alpha_{23}\left(\alpha_{22}^{-1}\left(\alpha_{1}\right)\right)
\end{aligned}
$$

and we also select the non-negative constant

$$
c_{L}=\alpha_{1}^{-1}\left(\sigma\left(\alpha_{12}\left(2 c_{L 1}\right)\right)\right)+\alpha_{1}^{-1}\left(\alpha_{12}\left(2 c_{L 2}\right)\right) \text {. }
$$


Fix a time $t_{0} \in I_{z, w}-(z, w)^{-1}(0)$ where $V^{z, w}, V_{1}^{z}$ and $V_{2}^{w}$ are differentiable and suppose that

$$
\left|\left(z\left(t_{0}\right), w\left(t_{0}\right)\right)\right| \geq c_{L} .
$$

It then suffices to show the inequality

$$
V^{\dot{z}, w}\left(t_{0}\right) \leq-\alpha_{3}\left(\left|\left(z\left(t_{0}\right), w\left(t_{0}\right)\right)\right|\right) .
$$

Using (50), we have three possible cases:

1) Suppose that $t_{0} \in D_{z}$, in particular, $z\left(t_{0}\right) \neq 0$. There exists $\epsilon_{1}>0$ such that

$$
V^{z, w}(t)=\sigma\left(V_{1}^{z}(t)\right),
$$

for all $t \in I_{z, w} \cap B_{\epsilon_{1}}\left(t_{0}\right)$. As a consequence,

$$
V^{\dot{z}, w}\left(t_{0}\right)=\dot{\sigma}\left(V_{1}^{z}\left(t_{0}\right)\right) \cdot \dot{V}_{1}^{z}\left(t_{0}\right) .
$$

Since

$$
V_{2}^{w}\left(t_{0}\right)<\sigma\left(V_{1}^{z}\left(t_{0}\right)\right),
$$

the inequality in (49) implies that

$$
2 \chi_{1}\left(\alpha_{21}^{-1}\left(V_{2}^{w}\left(t_{0}\right)\right)\right) \leq \alpha_{12}^{-1}\left(V_{1}^{z}\left(t_{0}\right)\right) .
$$

From property (7) of $V_{1}^{z}$ and $V_{2}^{w}$ it follows that

$$
\begin{aligned}
\chi_{1}\left(\left|w\left(t_{0}\right)\right|\right) & \leq \chi_{1}\left(\alpha_{21}^{-1}\left(V_{2}^{w}\left(t_{0}\right)\right)\right), \\
\alpha_{12}^{-1}\left(V_{1}^{z}\left(t_{0}\right)\right) & \leq\left|z\left(t_{0}\right)\right|
\end{aligned}
$$

and, therefore,

$$
\left|z\left(t_{0}\right)\right| \geq 2 \chi_{1}\left(\left|w\left(t_{0}\right)\right|\right) .
$$

On the other hand, (51) and (53) together with the properties of $\alpha_{1}$ imply

$$
\sigma\left(V_{1}^{z}\left(t_{0}\right)\right) \geq \sigma\left(\alpha_{12}\left(2 c_{L 1}\right)\right)
$$

and, hence,

$$
\left|z\left(t_{0}\right)\right| \geq 2 c_{L 1} .
$$

Summing this inequality with (55) leads to

$$
\left|z\left(t_{0}\right)\right| \geq \chi_{1}\left(\left|w\left(t_{0}\right)\right|\right)+c_{L 1}
$$

and, using (53) and (54), property (8) of $V_{1}^{z}$ implies:

$$
\begin{aligned}
V^{\dot{z}, w}\left(t_{0}\right) & \leq-\dot{\sigma}\left(\sigma^{-1}\left(V^{z, w}\left(t_{0}\right)\right)\right) \cdot \alpha_{13}\left(\alpha_{12}^{-1}\left(\sigma^{-1}\left(V^{z, w}\left(t_{0}\right)\right)\right)\right) \\
& \leq-\hat{\alpha}_{1}\left(\left|\left(z\left(t_{0}\right), w\left(t_{0}\right)\right)\right|\right) \\
& <-\alpha_{3}\left(\left|\left(z\left(t_{0}\right), w\left(t_{0}\right)\right)\right|\right) .
\end{aligned}
$$

2) Suppose that $t_{0} \in D_{w}$, in particular, $w\left(t_{0}\right) \neq 0$. The procedure in this case follows the same ideas. We have that:

$$
V^{z, w}\left(t_{0}\right)=V_{2}^{w}\left(t_{0}\right), \quad V^{\dot{z}, w}\left(t_{0}\right)=\dot{V}_{2}^{w}\left(t_{0}\right) .
$$

Inequality (49) implies

$$
2 \chi_{2}\left(\alpha_{11}^{-1}\left(V_{1}^{z}\left(t_{0}\right)\right)\right) \leq \alpha_{22}^{-1}\left(V_{2}^{w}\left(t_{0}\right)\right)
$$

and, together with assumption (51), we have

$$
\left|w\left(t_{0}\right)\right| \geq \chi_{2}\left(\left|z\left(t_{0}\right)\right|\right)+c_{L 2} .
$$

This in turn implies that

$$
\begin{aligned}
V^{\dot{z}, w}\left(t_{0}\right) & \leq-\alpha_{23}\left(\alpha_{22}^{-1}\left(V^{z, w}\left(t_{0}\right)\right)\right) \\
& \leq-\hat{\alpha}_{2}\left(\left|\left(z\left(t_{0}\right), w\left(t_{0}\right)\right)\right|\right) \\
& <-\alpha_{3}\left(\left|\left(z\left(t_{0}\right), w\left(t_{0}\right)\right)\right|\right) .
\end{aligned}
$$

3) Now suppose that $t_{0} \in D$ so that

$$
\begin{aligned}
V^{z, w}\left(t_{0}\right) & =\sigma\left(V_{1}^{z}\left(t_{0}\right)\right) \\
& =V_{2}^{w}\left(t_{0}\right) .
\end{aligned}
$$

In particular, both $z\left(t_{0}\right)$ and $w\left(t_{0}\right)$ differ from zero. Just as in the previous two cases, we have:

$$
\begin{aligned}
\dot{\sigma}\left(V_{1}^{z}\left(t_{0}\right)\right) \cdot \dot{V}_{1}^{z}\left(t_{0}\right) & <-\alpha_{3}\left(\left|\left(z\left(t_{0}\right), w\left(t_{0}\right)\right)\right|\right), \\
\dot{V}_{2}^{w}\left(t_{0}\right) & <-\alpha_{3}\left(\left|\left(z\left(t_{0}\right), w\left(t_{0}\right)\right)\right|\right) .
\end{aligned}
$$

As a consequence, there exists $\epsilon_{2}>0$ such that

$$
\begin{aligned}
\frac{\sigma\left(V_{1}^{z}(t)\right)-\sigma\left(V_{1}^{z}\left(t_{0}\right)\right)}{t-t_{0}} & <-\alpha_{3}\left(\left|\left(z\left(t_{0}\right), w\left(t_{0}\right)\right)\right|\right), \\
\frac{V_{2}^{w}(t)-V_{2}^{w}\left(t_{0}\right)}{t-t_{0}} & <-\alpha_{3}\left(\left|\left(z\left(t_{0}\right), w\left(t_{0}\right)\right)\right|\right),
\end{aligned}
$$

for all $t \in I_{z, w} \cap B_{\epsilon_{2}}\left(t_{0}\right)$. Then (56) leads to

$$
\frac{V^{z, w}(t)-V^{z, w}\left(t_{0}\right)}{t-t_{0}}<-\alpha_{3}\left(\left|\left(z\left(t_{0}\right), w\left(t_{0}\right)\right)\right|\right),
$$

for all $t \in I_{z, w} \cap B_{\epsilon_{2}}\left(t_{0}\right)$, which implies (52).

Finally, notice that the functions $\alpha_{1}, \alpha_{2}$ and $\alpha_{3}$ and the constant $c_{L}$ are all independent from the choice of $(z, w)$.

\section{REFERENCES}

[1] H. K. Khalil, Nonlinear systems, 3rd ed. New Jersey: Prentice Hall, 2002.

[2] T. Meurer, K. Graichen, and E.-D. Gilles, Eds., Control and observer design for nonlinear finite and infinite dimensional systems, ser. Lecture notes in control and information sciences. Berlin ; New York: Springer, 2005, no. 322.

[3] J.-P. Gauthier and I. Kupka, Deterministic observation theory and applications. Cambridge; New York: Cambridge University Press, 2001.

[4] H. Khalil, Nonlinear Control. New York: Pearson Education, Upper Saddle River, 2015.

[5] A. J. Krener and A. Isidori, "Linearization by output injection and nonlinear observers," Systems \& Control Letters, vol. 3, no. 1, pp. 47-52, Jun. 1983.

[6] H. Hammouri and J. Gauthier, "Bilinearization up to output injection," Systems \& Control Letters, vol. 11, pp. 139-149, 1988.

[7] G. Bornard, N. Couenne, and F. Celle, "Regular persistent observers for bilinear systems," Proceeding of the 29 international conference on nonlinear systems. New trend in nonlinear system theory. Vol 122 Springer Verlag, Jun. 1988.

[8] J. Gauthier, H. Hammouri, and S. Othman, "A simple observer for nonlinear systems. Applications to bioreactors," IEEE Transactions on Automatic Control, vol. 37, no. 6, pp. 875-880, Jun. 1992.

[9] H. K. Khalil and L. Praly, "High-gain observers in nonlinear feedback control," International Journal of Robust and Nonlinear Control, vol. 24, no. 6, pp. 993-1015, Apr. 2014.

[10] F. Esfandiari and H. K. Khalil, "Output feedback stabilization of fully linearizable systems," International Journal of Control, vol. 56, no. 5, pp. 1007-1037, Nov. 1992.

[11] L. K. Vasiljevic and H. K. Khalil, "Error bounds in differentiation of noisy signals by high-gain observers," Systems \& Control Letters, vol. 57, no. 10 , pp. 856-862, Oct. 2008.

[12] J. Ahrens and H. Khalil, "High-gain observers in the presence of measurement noise: A switched-gain approach," Automatica, vol. 45, no. 4, pp. 936-943, Apr. 2009.

[13] N. Boizot, E. Busvelle, and J.-P. Gauthier, "An adaptive high-gain observer for nonlinear systems," Automatica, vol. 46, no. 9, pp. 1483 1488, Sep. 2010.

[14] A. Alessandri and A. Rossi, "Time-varying increasing-gain observers for nonlinear systems," Automatica, vol. 49, no. 9, pp. 2845-2852, Sep. 2013.

[15] S. Suranthiran and S. Jayasuriya, "Signal Conditioning With MemoryLess Nonlinear Sensors," Journal of Dynamic Systems, Measurement, and Control, vol. 126, no. 2, p. 284, 2004. 
[16] C. W. De Silva, Sensors and actuators: control systems instrumentation. Boca Raton, FL: CRC Press, 2007.

[17] M. Arcak, H. Gorgun, L. Pedersen, and S. Varigonda, "A Nonlinear Observer Design for Fuel Cell Hydrogen Estimation," IEEE Transactions on Control Systems Technology, vol. 12, no. 1, pp. 101-110, Jan. 2004.

[18] A. Tekalp and G. Pavlovic, "Image restoration with multiplicative noise: incorporating the sensor nonlinearity," IEEE Transactions on Signal Processing, vol. 39, no. 9, pp. 2132-2136, Sep. 1991.

[19] A. Rush, "Nonlinear sensors impact digital imaging," Electronics Engineer, 1998.

[20] C. O. Nwagboso, Automotive Sensory Systems. Dordrecht: Springer Netherlands, 1993.

[21] M. Kothari, J. G. Webster, W. J. Tompkins, J. J. Wertsch, and P. Bach y Rita, "Capacitive sensors for measuring the pressure between the foot and shoe," Proceedings of the Annual International Conference of the IEEE Engineering in Medicine and Biology Society, pp. 805-806, 1988.

[22] K. Reif, F. Sonnemann, and R. Unbehauen, "Nonlinear state observation using H_infinity-filtering Riccati design," IEEE Transactions on Automatic Control, vol. 44, no. 1, pp. 203-208, Jan. 1999.

[23] M. Arcak and P. Kokotović, "Nonlinear observers: a circle criterion design and robustness analysis," Automatica, vol. 37, no. 12, pp. 1923 1930, Dec. 2001.

[24] X. Fan and M. Arcak, "Observer design for systems with multivariable monotone nonlinearities," Systems \& Control Letters, vol. 50, no. 4, pp. 319-330, Nov. 2003.

[25] B. Açıkmeşe and M. Corless, "Observers for systems with nonlinearities satisfying incremental quadratic constraints," Automatica, vol. 47, no. 7, pp. 1339-1348, Jul. 2011.

[26] J. Lei and H. K. Khalil, "High-gain observers in the presence of sensor nonlinearities," in 2017 American Control Conference (ACC). Seattle, WA, USA: IEEE, May 2017, pp. 3282-3287.

[27] G. Besançon, Ed., Nonlinear observers and applications, ser. Lecture notes in control and information sciences. Berlin ; New York: Springer, 2007, no. 363.

[28] R. Marino and P. Tomei, "Global adaptive observers for nonlinear systems via filtred transformations," IEEE Trans. on Automatic Control, vol. 37, no. 8, 1992.

[29] E. D. Sontag, "Smooth stabilization implies coprime factorization," IEEE transactions on automatic control, vol. 34, no. 4, pp. 435-443, 1989.

[30] S. N. Dashkovskiy, D. V. Efimov, and E. D. Sontag, "Input to state stability and allied system properties," Automation and Remote Control, vol. 72, no. 8, pp. 1579-1614, Aug. 2011.

[31] E. D. Sontag and Y. Wang, "Output-to-state stability and detectability of nonlinear systems," Systems \& Control Letters, vol. 29, no. 5, pp. 279-290, Feb. 1997.

[32] H. Shim and D. Liberzon, "Nonlinear Observers Robust to Measurement Disturbances in an ISS Sense," IEEE Transactions on Automatic Control, vol. 61, no. 1, pp. 48-61, Jan. 2016.

[33] H. Shim, J. Seo, and A. Teel, "Nonlinear observer design via passivation of error dynamics," Automatica, vol. 39, no. 5, pp. 885-892, May 2003.

[34] A. Alessandri, "Observer design for nonlinear systems by using Inputto-State Stability," in 43rd IEEE Conference on Decision and Control (CDC). Nassau, Bahamas: IEEE, 2004, pp. 3892-3897 Vol.4.

[35] Z.-P. Jiang, I. M. Mareels, and Y. Wang, "A Lyapunov formulation of the nonlinear small-gain theorem for interconnected ISS systems," Automatica, vol. 32, no. 8, pp. 1211-1215, 1996.

[36] H. Hammouri and M. Nadri, "An observer design for a class of implicit systems," Systems \& Control Letters, vol. 62, no. 3, pp. 256-261, Mar. 2013.

[37] H. F. Grip, A. Saberi, and T. A. Johansen, "Observers for interconnected nonlinear and linear systems," Automatica, vol. 48, no. 7, pp. 1339-1346, Jul. 2012.

[38] F. González de Cossío, M. Nadri, and P. Dufour, "Observer design for nonlinear systems with implicit ouput," in 2018 IEEE Conference on Decision and Control (CDC). Miami Beach, FL: IEEE, Dec. 2018, pp. 2170-2175.

[39] E. D. Sontag and Y. Wang, "On characterizations of the input-to-state stability property," Systems \& Control Letters, vol. 24, no. 5, pp. 351359, Apr. 1995.

[40] H. A. Edwards, Y. Lin, and Y. Wang, "On input-to-state stability for time varying nonlinear systems," in Proceedings of the 39th IEEE Conference on Decision and Control, vol. 4. Sydney, Australia: IEEE, 2000, pp. 3501-3506.

[41] C. M. Kellett, "A compendium of comparison function results," Mathematics of Control, Signals, and Systems, vol. 26, no. 3, pp. 339-374, Sep. 2014.
[42] R. Freeman and P. Kokotovic, Robust Nonlinear Control Design: StateSpace and Lyapunov Techniques, ser. Modern Birkhäuser Classics. Birkhäuser Boston, 2008.

[43] Z.-P. Jiang, A. R. Teel, and L. Praly, "Small-gain theorem for ISS systems and applications," Mathematics of Control, Signals and Systems, vol. 7, no. 2, pp. 95-120, 1994.

[44] E. D. Sontag and Y. Wang, "On characterizations of input-to-state stability with respect to compact sets," in Nonlinear Control Systems Design 1995. Elsevier, 1995, pp. 203-208.

[45] S. Dashkovskiy, B. S. Rüffer, and F. R. Wirth, "An ISS small gain theorem for general networks," Mathematics of Control, Signals, and Systems, vol. 19, no. 2, pp. 93-122, May 2007.

[46] J. Cronin, Ordinary Differential Equations: Introduction and Qualitative Theory, Third Edition, 3rd ed. Boca Raton: CRC Press, Dec. 2007.

[47] H. Hammouri and J. de Leon Morales, "Observer synthesis for stateaffine systems," in 29th IEEE Conference on Decision and Control. Honolulu, HI, USA: IEEE, 1990, pp. 784-785 vol.2.

[48] G. Besançon, G. Bornard, and H. Hammouri, "Observer Synthesis for a Class of Nonlinear Control Systems," European Journal of Control, vol. 2, no. 3, pp. 176-192, 1996.

[49] F. González de Cossío, M. Nadri, and P. Dufour, "Optimal observer design for disturbed state affine systems," in 2018 Annual American Control Conference (ACC). Milwaukee, WI: IEEE, Jun. 2018, pp. 2733-2738.

[50] Y. Lin, E. Sontag, and Y. Wang, "A Smooth Converse Lyapunov Theorem for Robust Stability," SIAM J. Control and Optimization, vol. 34, no. 1, Jan. 1996.

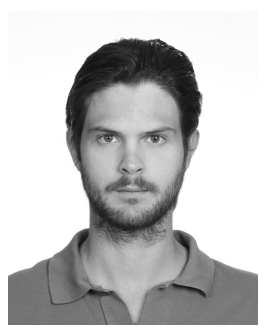

Francisco González de Cossío was born in Mexico City, Mexico in 1989. He received the B.S. degree in mathematics from Universidad Nacional Autónoma de México, Mexico City, Mexico, in 2013 and the M.S. degree in mathematics from Central European University, Budapest, Hungary, in 2015. He is currently a Ph.D. candidate in automatic control at Université Claude Bernard Lyon 1, Villeurbanne, France.

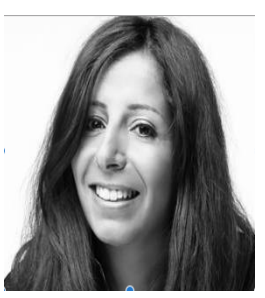

Madiha Nadri received the M.S. degree in automatic control from Université Claude Bernard Lyon 1, Villeurbanne, France, in 1997. In 2001, she received the Ph.D. degree from the same university.

After working one year as Lecturer, she joined the Division of Applied Mathematics and Process Control at IFP Energies Nouvelles as a Research Assistant. Since 2005, she is an Assistant Professor in automatic control at the Department of Electrical and Chemical Engineering, Université Claude Bernard Lyon 1. Her research interests include state estimation problems, nonlinear observers, identification and Lyapunov stability of nonlinear systems, especially in biological systems and automotive applications.

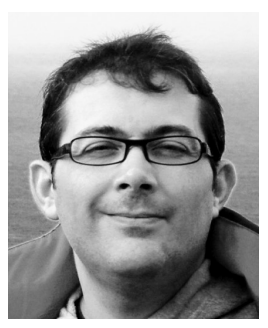

Pascal Dufour is Associate Professor in automatic control with Université Claude Bernard Lyon 1 (UCBL1), Villeurbanne, France. After receiving the Ph.D. degree from UCBL1 in 2000, he was a PostDoctoral Fellow with the University of Delaware, Newark, DE, USA, from 2000 to 2001. He joined UCBL1 in 2001 as a permanent staff. His current research interests include model-based control approach, model predictive control, observer-based closed loop identification, and optimization techniques, with applications in process system engineering (Rankine based waste heat recovery system for trucks, heat pumps control, drying, lyophilization, systems with heat and mass transfer). 